\title{
Pollen and spores from surface samples in the campos region of Uruguay and their paleoecological implications
}

\author{
Dominique Mourelle ${ }^{1 \star}$ and Aldo R. Prieto ${ }^{2}$
}

Received: April 7, 2016

Accepted: May 25, 2016

\begin{abstract}
In this study we describe and illustrate pollen and spores that have been identified as significant in modern and fossil samples from the campos region of Uruguay. We provide new information about modern pollen assemblages and their relationship to the vegetation types of this region. We discuss the taxonomic limitations of pollen and spores and their representation in both modern and fossil samples. We highlight the importance of identifying both silent and key indicator taxa for making accurate paleoecological interpretations. We also emphasize the importance of knowing the pollination strategies of parental plants because many of them are zoophilous, and so small changes in the proportions of their pollen grains in fossil assemblages could reflect important changes in vegetation. This study presents a practical approach to paleoecological research, which not only produces robust results in studies of the campos region, but can be applied to other grassland ecosystems, including those in temperate regions.
\end{abstract}

Keywords: Holocene sequences, key indicator taxa, modern pollen-vegetation relationships, pollen and spores descriptions, silent taxa

\section{Introduction}

Pollen analysis, the study of fossil pollen and spores, is the principal technique for long-term vegetation reconstructions (Seppä 2007), and it has provided fundamental advances in the understanding of the processes and mechanisms of ecological change (e.g. Jackson 2007; Rull 2010; Willis et al. 2010). However, pollen records do not directly reflect plant abundance due to several factors such as differences in pollen production, dispersal and preservation of taxa (Prentice 1988), which cause that taxa may be either over-represented or under-represented in pollen samples. Species with anemophilous pollination, which produce high quantities of pollen grains, are frequently overrepresented in pollen assemblages while zoophilous, with low pollen productivity, are under-represented (Fægri \& Iversen 1989). Palynologist must work with an incomplete representation of taxa because many floristically important families contribute little or no pollen to pollen rain (Bush et al. 2001).

One of the principles on which pollen analysis is based is the possibility of identifying different pollen and spores according to their morphology (Fægri \& Iversen 1989). There are some difficulties in studying modern pollen-vegetation relationships in Río de la Plata grasslands (RPG) (Southern South America) related to the limited pollen taxonomic resolution of their most abundant parental plants, such as Poaceae, Cyperaceae, Asteraceae subf. Asteroideae and some genera of the Apiaceae family (Tonello \& Prieto 2008; Mourelle \& Prieto 2012). However, there are a great number

\footnotetext{
${ }^{1}$ Centro Universitario Regional Este, Ruta Nacional n 9, 27000, Ciudad de Rocha, Uruguay

${ }^{2}$ Laboratorio de Paleoecología y Palinología, Facultad de Ciencias Exactas y Naturales, Universidad Nacional de Mar del Plata, Dean Funes 3350 , San Juan, 7600, Mar del Plata, Argentina

* Corresponding author: domodica@gmail.com
} 
of minor taxa that allow the qualitative and quantitative analysis of pollen-vegetation relationships.

In particular, palynological analyses in the campos region of Uruguay revealed that pollen and spores in surface samples (Mourelle \& Prieto 2012) and Holocene records (Iriarte 2006; García-Rodríguez et al. 2010; Mourelle et al. 2015a; b) are very diverse. However, even though several pollen and spores atlas (e.g. Bauermann et al. 2013; Radaeski et al. 2014) and catalogues (e.g. Neves \& Bauermann 2004; Macedo et al. 2009; Evaldt et al. 2013; Masetto \& Lorscheitter 2014) in the campos region of Rio Grande do Sul have been published during the last decades, there are no morphological descriptions and illustrations for the most frequent and abundant pollen and spores that appeared in surface and fossil samples from the campos region of Uruguay.

For this reason we propose the following aims in this study: (1) to describe and illustrate the main pollen and spores from surface samples from the campos region of Uruguay; (2) to provide new information about modern pollen assemblages and their relationship to the vegetation types of this region; and (3) to discuss the taxonomic limitations of pollen and spores and their representation in both modern and fossil samples, and provide a practical approach to obtaining robust results for paleoecological research in the campos region.

\section{Study area}

RPG extend between $28^{\circ}$ and $38^{\circ} \mathrm{S}$ latitude, covering approximately $700,000 \mathrm{~km}^{2}$ of eastern Argentina, Uruguay and southern Rio Grande do Sul (Brazil) (Fig. 1) and is characterized by the dominance of grasses and other herbs. Based on geomorphological, hydrological and edaphic features and their link with natural vegetation and land use, the RPG were divided by León (1991) into the pampas in the eastern Argentina, and the campos in Uruguay and southern Rio Grande do Sul (Brazil). The presence of woody species and grasses with $\mathrm{C} 4$ metabolism gradually diminishes from north to south, one of the facts that allowed the division of this sub-region in southern and northern campos (León 1991) (Fig. 1). Most of these shrubby and arboreal species in Uruguay reach their southern distribution range and so they are absent in the pampas. These RPG are climatically determined (Bilenca \& Miñarro 2004). The climate is temperate, with less extreme conditions than those in other regions located at similar latitudes due to the Atlantic Ocean moderating effect (Soriano 1991).

Natural grasslands are the predominant vegetation type of the campos region (Fig. 1) and are extended all over Uruguay (ca. $77 \%$ of the territory), in flat and gentlyrolling relief, hills, gentle hills and low mountains (Puerto

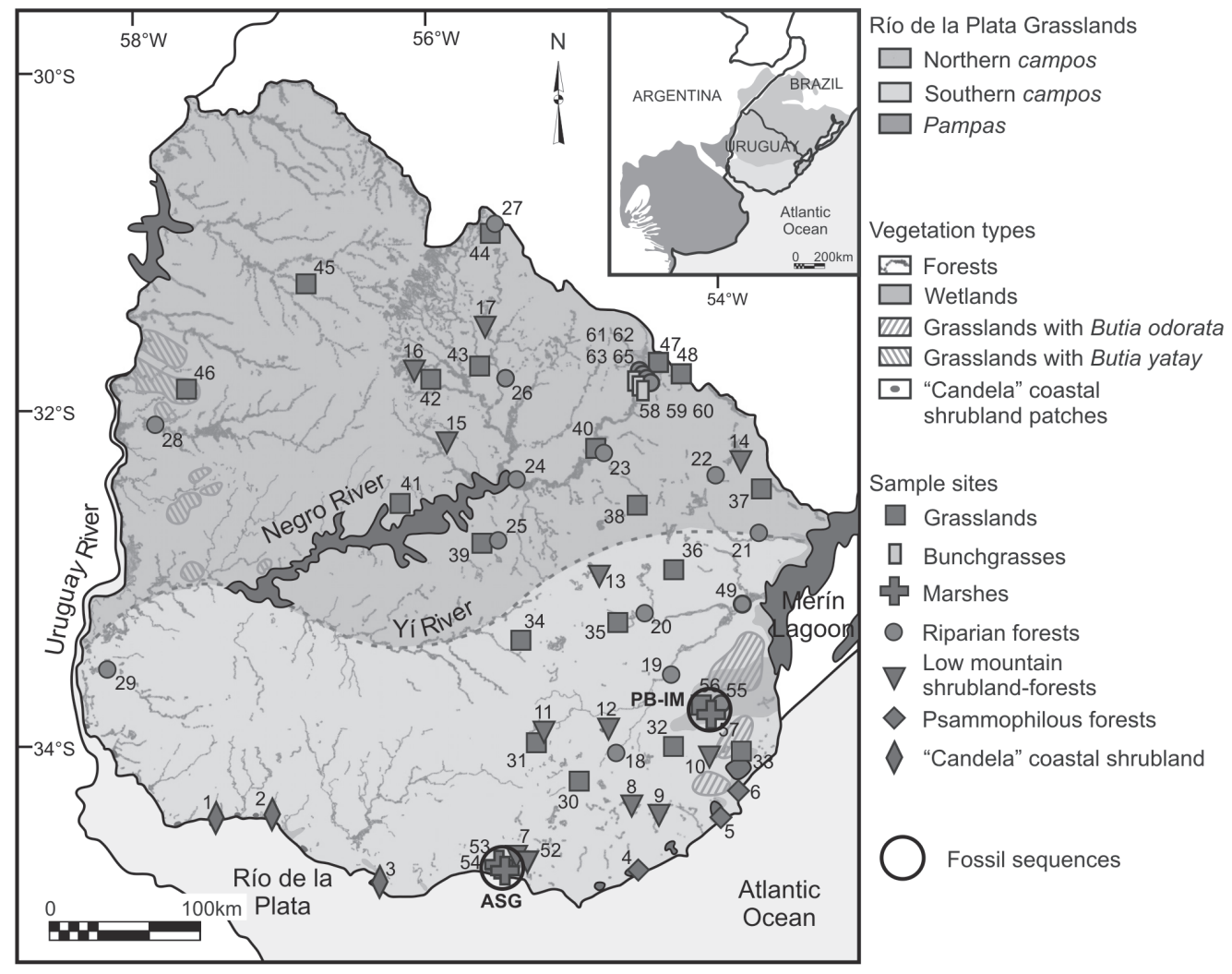

Figure 1. Uruguay map showing the main vegetation types and the location of the 62 surface samples (modified from Mourelle \& Prieto 2012) and the Holocene sequences (ASG, Arroyo Solís Grande, Mourelle et al. 2015a; PB-IM, Paso Barranca-India Muerta, Mourelle et al. 2015b). The map scale does not allow the representation of bunchgrasses. Inset map: Río de la Plata grasslands (León 1991). 
1987; Lezama et al. 2011) (Tab. 1). Woody vegetation covers approximately $4.3 \%$ of the territory (Petraglia \& Dell'Acqua 2006) and is composed of about 300 species of trees and shrubs, characteristic elements of forests and shrublands, respectively (Brussa \& Grela 2007; Haretche et al. 2012). Forests, shrublands and other herbs-dominated vegetation (not grasslands) develop locally in environments with particular edaphic conditions that define each different vegetation type (Tab. 1).

After the European settlement, the campos region of Uruguay has progressively become one of the most important areas of livestock and grain production in the world (Bilenca \& Miñarro 2004). Furthermore, the expansion of cropland areas, the introduction of exotic grass species and their associated weeds, and more recently the substitution of grasslands for exotic forest plantations, deeply modified the original landscape.

\section{Materials and methods}

\section{Selection of pollen and spores taxa}

Described and illustrated taxa were selected from 62 surface samples from the campos region of Uruguay based upon two criteria: (1) their abundance and frequency in surface samples and (2) their occurrence in fossil records previously published (Mourelle et al. 2015a; b) (Fig. 1). A total of 46 surface samples were previously studied (Mourelle $\&$ Prieto 2012) and 16 further samples were collected and incorporated in this study (additional data are provided in Mourelle 2015). All samples were collected following Adam \& Mehringer (1975) sampling strategy and they correspond to seven different vegetation types: grasslands, riparian forests, low-mountain shrublands-forests; psammophilous forests, "Candela" coastal shrublands, bunchgrasses and marshes (Fig. 1). The sample sites are principally located in the eastern part of Uruguay because: (1) it concentrates a larger number of different vegetation types than the western part; (2) it has minor human disturbance; and (3) it is where the fossil pollen sequences studied are located (Fig. 1).

\section{Laboratory work}

Surface samples were dried at $60^{\circ} \mathrm{C}$ for $24 \mathrm{~h}$. Subsamples of 5 to $15 \mathrm{~g}$ dry sediment were used for pollen analysis. Sediment samples were sieved trough a $110 \mathrm{~mm}$ screen to remove coarse particles and then processed according to standard palynological techniques including warm $\mathrm{KOH}$, $\mathrm{HCl}$, heavy-liquid separation with $\mathrm{ZnCl}_{2}, \mathrm{HF}$ and acetolysis (Fægri \& Iversen 1989).

\section{Pollen and spores descriptions}

Standard morphological descriptions of palynomorphs (pollen and spores) follow Punt et al. (2007) and Hesse et al. (2009). Pollen shape classes are described according to ratios of their polar and equatorial axes ( $\mathrm{P}$ :E ratios) in agreement with Erdtman (1971). Descriptions were checked using the pollen and spores reference collection of the Laboratory of Palaeoecology and Palynology, Universidad Nacional de Mar del Plata, Argentina. Identifications were supplemented by published atlas and photographs (Markgraf \& D'Antoni 1978; Prieto \& Quattrocchio 1993; Pire et al. 1998; 2001; 2006; Bauermann et al. 2013).

Under the light microscope, at least 20 pollen grains or spores per each taxonomic entity were measured to elaborate each morphological description, using a 100x oil immersion objective. Measurements were made using an ocular micrometer in the microscope. All images were taken by a Nikon Coolpix P3 digital camera. Pollen slide preparations have been deposited in the palynological collection at the Laboratory of Paleoecology and Palynology, Universidad Nacional de Mar del Plata, Argentina.

For pollen and spores nomenclature we followed some conventions recommended by Birks \& Birks (1980): (1) if pollen or spores types are known to be produced by only one species they are named after this species (e.g. PHYLLANTHus SELLOWIANUS); (2) if a complete family produces pollen or spores that cannot be morphologically distinguished, the type is named after the family (e.g. POACEAE); (3) if the pollen or spores morphological category is known to be produced by two taxa, then it is named after these two taxa (e.g. LithraeA/Schinus); (4) if three or more plant taxonomical entities are known to produce the same morphological pollen or spore type, the suffix "type" is added after the taxonomical name (e.g. AlternantHERA-TYPE). Since there are significant differences between the concepts of "plant taxa" and "palynomorphological types" and in order to provide the necessary clarity on the nomenclature, names of pollen and spores were put in SMALL CAPITALS and refrain from using italics (Klerk \& Joosten 2007).

Pollen were classified according to the classification system APG III (2009) and spores in accordance with the Missouri Botanical Garden (MOBOT 2016). The palynological terminology follows Punt et al. (2007) and Hesse et al. (2009).

On the other hand, pollen and spores from surface samples were counted using a microscope with $1000 \mathrm{x}$ magnification. A minimum of 300 pollen grains were counted, including pollen from herbs and aquatic herbs (herbaceous pollen), shrubs (shrubby pollen), trees (arboreal pollen) and climbers, and they represent the total pollen sum. Due to the over-representation of POACEAE in grassland samples, 300 pollen grains excluding POACEAE were counted in order to obtain a good representation of the subordinated taxa. Pteridophytes and bryophytes were counted and expressed as percentages of the total palynomorphs sum. Palynomorph percentages were calculated and diagrams were drawn using TGView 2.0.4 program (Grimm 2004). 
Table 1. Description of the vegetation types (Chebataroff 1960; Puerto 1969; 1987; León 1991; Alonso-Paz \& Bassagoda 1999; 2002; Fagúndez \& Lezama 2005; Brussa \& Grela 2007; Lezama et al. 2011) and the characteristic pollen and spores from modern surface samples in the campos region of Uruguay.

\begin{tabular}{|c|c|c|c|}
\hline Vegetation type & Habitat & Vegetation frequent taxa & Surface samples: characteristic taxa \\
\hline \multicolumn{4}{|l|}{ REGIONAL } \\
\hline Grasslands & $\begin{array}{l}\text { Flat and gently-rolling } \\
\text { relief, hills, gentle hills } \\
\text { and low mountains }\end{array}$ & $\begin{array}{l}\text { Stipa spp., Aristida spp., Andropogon spp., Briza spp., Erianthus } \\
\text { spp., Piptochaetium spp., Paspalum spp., Axonopus spp., } \\
\text { Panicum spp. (Poaceae), Carex spp., Cyperus spp., Eleocharis } \\
\text { spp., Kyllinga spp., Bulbostylis spp. (Cyperaceae), Baccharis } \\
\text { spp., Eupatorium spp. (Asteraceae), Eryngium spp. (Apiaceae), } \\
\text { Trifolium polymorphum, Adesmia bicolor (Fabaceae), Dichondra } \\
\text { sericea (Convolvulaceae), Oxalis spp. (Oxalidaceae), Glandularia } \\
\text { spp. (Verbenaceae). Butia odorata and Butia yatay (Arecaceae) in } \\
\text { some grasslands. }\end{array}$ & 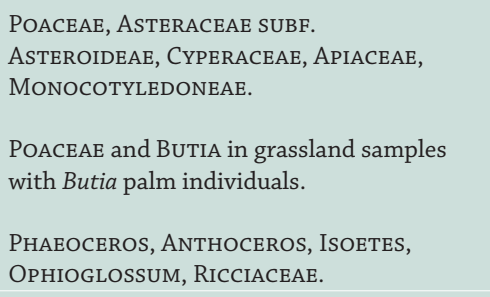 \\
\hline \multicolumn{4}{|l|}{ LOCAL } \\
\hline \multirow[t]{2}{*}{ Marshes } & $\begin{array}{l}\text { Freshwater: frequently } \\
\text { inland, in soils with } \\
\text { permanent high } \\
\text { groundwater }\end{array}$ & $\begin{array}{l}\text { Emergent herbs: Echinodorus grandiflorus, Sagittaria } \\
\text { montevidensis (Alismataceae), Canna glauca (Cannaceae), } \\
\text { Schoenoplectus californicus, Scirpus giganteus (Cyperaceae), } \\
\text { Thalia geniculata, T. multiflora (Marantaceae), Typha } \\
\text { domingensis, Zizaniopsis bonariensis (Poaceae). } \\
\text { Floating herbs: Pistia stratiotes, Lemna spp. (Araceae), Ludwigia } \\
\text { peploides (Onagraceae), Azolla spp., Salvinia spp. (Salviniaceae). } \\
\text { Submerged herbs: Myriophyllum spp. (Haloragaceae), } \\
\text { Potamogeton spp. (Potamogetonaceae). }\end{array}$ & $\begin{array}{l}\text { PoAceae, Cyperaceae, Asteraceae subf. } \\
\text { Asteroideae. }\end{array}$ \\
\hline & $\begin{array}{l}\text { Brackish: at the lower } \\
\text { reaches of major rivers } \\
\text { that flow into the } \\
\text { Atlantic Ocean and the } \\
\text { Río de la Plata estuary }\end{array}$ & Juncus acutus (Juncaceae), Spartina densiflora (Poaceae). & \\
\hline Bunchgrasses & $\begin{array}{l}\text { Grassland-forest } \\
\text { or grassland-marsh } \\
\text { boundary }\end{array}$ & $\begin{array}{l}\text { Cortaderia selloana, Erianthus angustifolius, Panicum prionitis, } \\
\text { Paspalum quadrifarium (Poaceae). }\end{array}$ & $\begin{array}{l}\text { PoAcEAe Apiaceae AsteraceAe subf. } \\
\text { AsteroideAe CyperaceAe. }\end{array}$ \\
\hline $\begin{array}{l}\text { Low mountain } \\
\text { shrublands-forests }\end{array}$ & Hills, rocky slopes & $\begin{array}{l}\text { Trees: Lithraea molleoides, Schinus lentiscifolia, S. longifolia, S. } \\
\text { molle (Anacardiaceae), Celtis ehrenbergiana (Cannabaceae), } \\
\text { Myrsine coriacea, M. laetevirens (Primulaceae), Blepharocalyx } \\
\text { salicifolius, Myrcianthes cisplatensis (Myrtaceae), Scutia buxifolia } \\
\text { (Rhamnaceae). } \\
\text { Shrubs: Baccharis spp., Heterothalamus alienus (Asteraceae), } \\
\text { Croton spp. (Euphorbiaceae), Mimosa spp. (Fabaceae), Colletia } \\
\text { paradoxa (Rhamnaceae), Dodonaea viscosa (Sapindaceae), } \\
\text { Daphnopsis racemosa (Thymelaeaceae). }\end{array}$ & 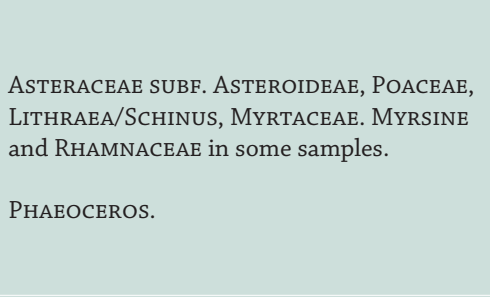 \\
\hline $\begin{array}{l}\text { Psammophilous } \\
\text { forests }\end{array}$ & $\begin{array}{l}\text { Sandy soils in coastal } \\
\text { zones }\end{array}$ & $\begin{array}{l}\text { Trees: Lithraea molleoides, S. lentiscifolia, S. longifolia, S. } \\
\text { molle (Anacardiaceae), Celtis ehrenbergiana (Cannabaceae), } \\
\text { Myrsine coriacea, M. laetevirens (Primulaceae), Scutia buxifolia } \\
\text { (Rhamnaceae). } \\
\text { Shrubs: Baccharis spp., Heterothalamus alienus (Asteraceae), } \\
\text { Croton spp. (Euphorbiaceae), Mimosa spp. (Fabaceae), Colletia } \\
\text { paradoxa (Rhamnaceae), Dodonaea viscosa (Sapindaceae), } \\
\text { Daphnopsis racemosa (Thymelaeaceae). }\end{array}$ & 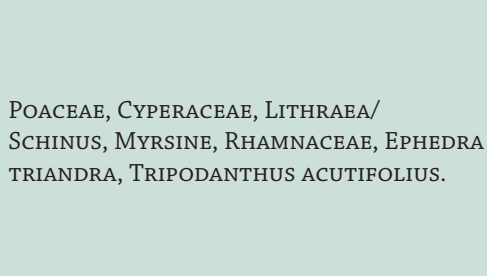 \\
\hline $\begin{array}{l}\text { "Candela" coastal } \\
\text { shrublands }\end{array}$ & $\begin{array}{l}\text { Mobile and semi-fixed } \\
\text { dunes in coastal zones }\end{array}$ & $\begin{array}{l}\text { Dodonaea viscosa (Sapindaceae), Baccharis spp., Eupatorium } \\
\text { buniifolium (Asteraceae). }\end{array}$ & $\begin{array}{l}\text { DODONAEA Viscosa, PoAceae, Asteraceae } \\
\text { SUbF. ASTERoIdEAE. }\end{array}$ \\
\hline
\end{tabular}




\section{Numerical analyses}

Numerical analyses were performed in order to determine whether the vegetation types are associated with characteristic pollen spectra from which the parent vegetation can be identified. Pollen types were selected if the mean value of each type in percentage was higher than $2 \%$ in at least one sample (spores were excluded due to their local over-representation). The percentage data were square root transformed prior to numerical analyses in order to stabilize their variances. Pollen samples were classified into groups using Cluster Analysis unconstrained (Birks \& Gordon 1985) with Euclidean distance (Overpeck et al. 1985), performed with TGView 2.0.4 program (Grimm 2004).

In order to evaluate the woody pollen dispersal from riparian forests to grasslands, seventeen grassland surface samples were analyzed considering (1) their distance to the riparian forests and (2) their woody pollen content. Grassland samples were selected only if a riparian forest represented the closest vegetation type. Woody pollen dispersal was assessed by plotting woody pollen content in grassland surface samples (as percentages) versus the distance to the nearest riparian forest (in meters). The power trend line has been added to the scatter plot.

\section{Results}

From the 62 surface samples, the total palynomorph sum reached a total of 86 pollen and spores related to the natural vegetation of the campos region of Uruguay. Only 35 pollen grains and 8 spores were the most representative in terms of abundance and frequency (Figs. 1, 2; Tab. 1). They are described in Tables 2 and 3 and illustrated in figures 3,4 and 5 .

Unconstrained Cluster Analysis shows distinctive palynological assemblages which characterized the main vegetation types of the campos region of Uruguay (Fig. 6). Seven vegetation types in tree groups of pollen assemblages were identified. Group I represents grasslands (Ia), bunchgrasses (Ib) and marshes (Ic). Group II comprises samples from "Candela" (Dodonaea viscosa) coastal shrublands (IIa) and from low-mountain shrublands-forests and psammophilous forests (IIb). Group III represents riparian forests.

The power trend line clearly demonstrates that the woody pollen from riparian forests shows very low dispersion capacity to grasslands. R-squared value of 0.86 indicates a very good fitness of the curve to the data set (Fig. 7).

The 43 palynomorph from surface samples described in this study were also the most abundant and frequent in the Arroyo Solís Grande (ASG) and Paso Barranca-India Muerta (PB-IM) fossil sequences (Mourelle et al. 2015a; b) (Fig. 8). They represent $52 \%$ and $58 \%$ of the pollen types that compose the total palynomorphs identified in ASG and PB-IM sequences, respectively (additional data are provided in Mourelle 2015).

\section{Discussion}

The importance of these pollen and spores, in both modern and fossil samples, is discussed below and represents the basis of this study, as well as it provides a practical approach to obtaining reliable results.

\section{Modern pollen-vegetation relationship}

Pollen analyses from modern surface samples revealed the good relation between modern pollen assemblages and the principal vegetation types of the campos region of Uruguay, mainly related to either the dominance of some taxa (e.g. PoAceAe in grasslands; DodonAeA VISCOSA in "Candela" coastal shrublands) or to the presence of key indicator taxa (e.g. hydrophilous woody taxa in riparian forests) (Figs. 2, 6). However, the low taxonomic resolution of some pollen taxa (e.g. AsteraceAe SUbF. AsteroideAe, Fig. 5U-V; Myrtaceae, Fig. 5A; Rhamnaceae, Fig. 4V) and the absence of pollen from some species that develop exclusively in some vegetation types (e.g. Sideroxylon obtusifolium, Rollinia maritima and Varronia curassavica in psammophilous forests; Juncus acutus in brackish marshes) make it difficult to differentiate some vegetation types only by the palynological assemblages. As a consequence, a loss of ecological information is expected.

Grassland pollen assemblages are dominated by herbs (ca. 80\%; Group Ia, Fig. 6) while riparian forests are dominated by woody pollen taxa (ca. 70\%; Group III, Fig. 6). Because riparian forests are dense, pollen dispersed from grasslands cannot easily enter into these forests. Consequently, grasslands pollen signal, regionally dominant, is under-represented in the pollen spectra from samples taken inside the riparian forests, although they consist mostly of trees and shrubs with zoophilous pollination (Tab. 3). Since other forests and scrublands of the campos region are less dense, the grassland regional pollen signal is better represented in such pollen assemblages, which are co-dominated by herbs, trees and shrubs (Group II, Fig. 6).

\section{Grasslands}

PoAceae (Fig. 4F) and Cyperaceae (Fig. 4E) dominate grassland pollen assemblages (Fig. 2; Tab. 1). Both families have anemophilous pollination and they represent the most abundant taxa in this vegetation type such as Stipa, Aristida, Paspalum, Piptochaetium and Briza, and Carex, Cyperus, Eleocharis, Kyllinga and Bulbostylis, respectively. Despite Asteraceae family is insect pollinated, ASTERACEAE SUBF. ASTEROIDEAE accompanies the herbs dominance in these pollen assemblages and reflects the high frequency of this 


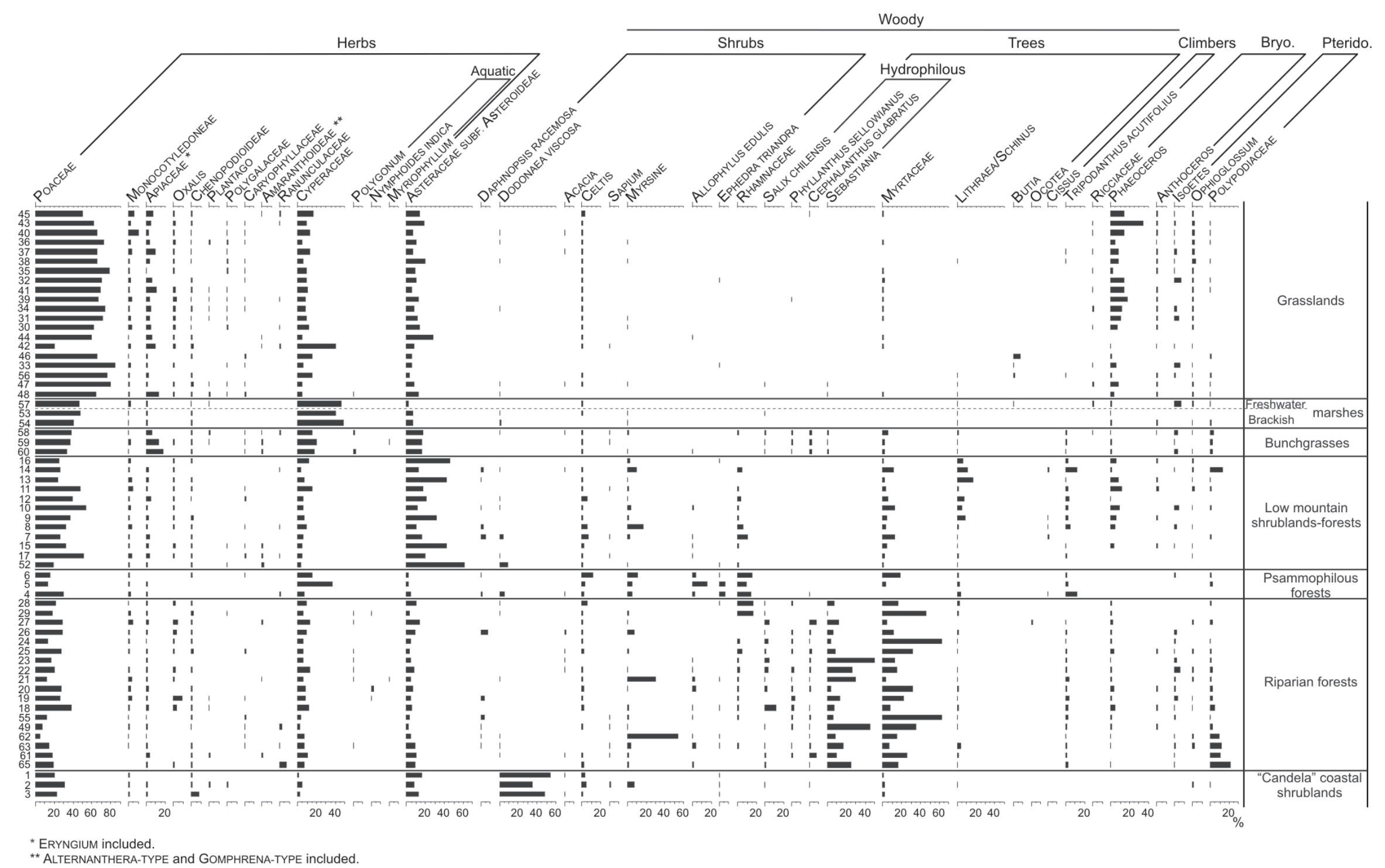

Figure 2. Pollen diagram of the 62 surface soil samples in percentages, ordered according to their vegetation types. Bryo.: Bryophytes; Pterido.: Pteridophytes. 
Table 2. Morphological descriptions of spores from the campos region of Uruguay. DF: distal face; PF; proximal face. PV: polar view; EqV: equatorial view. PD: polar diameter; EqD: equatorial diameter.

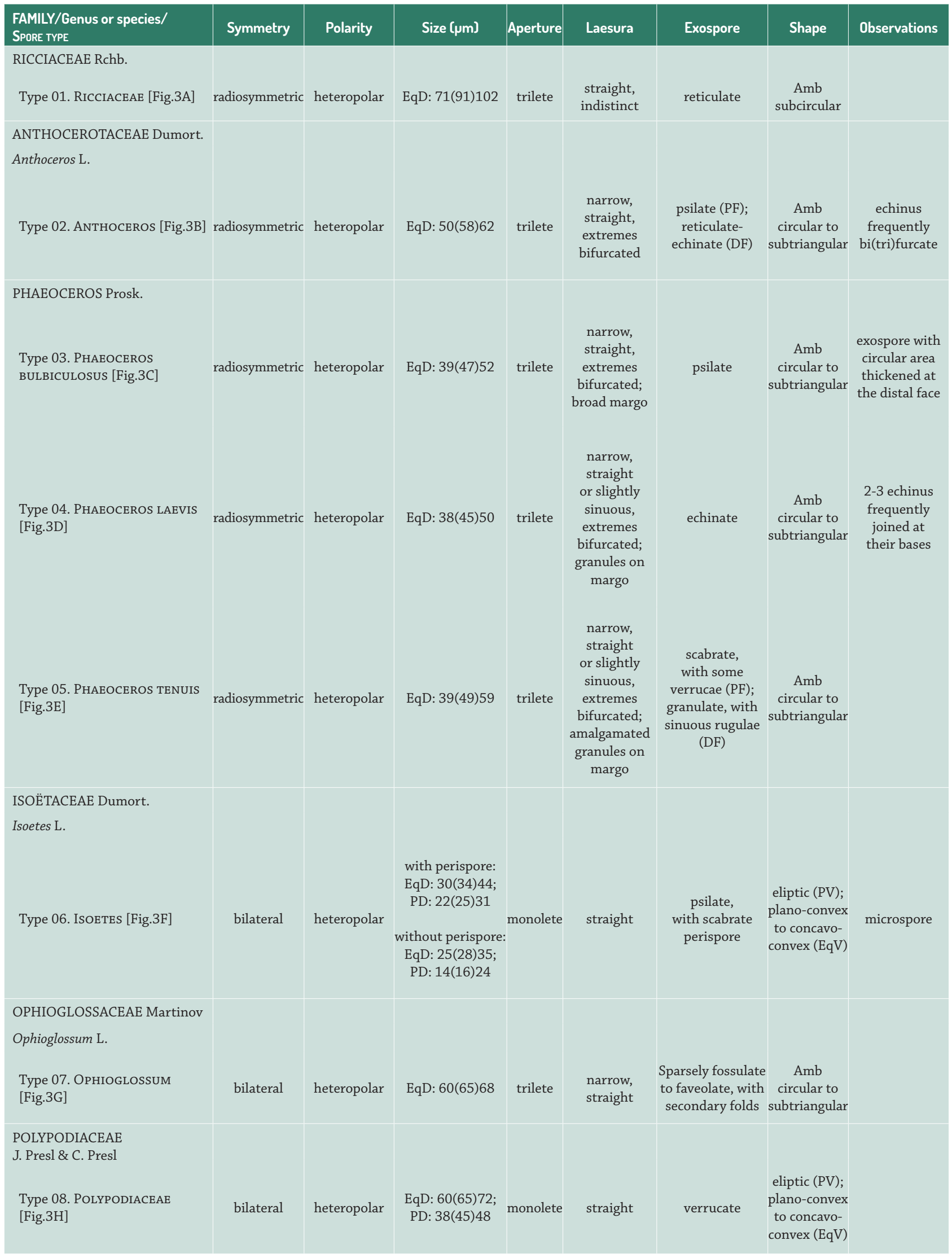


Table 3. Morphological descriptions of pollen from the campos region of Uruguay. PV: polar view; EqV: equatorial view. PD: polar diameter; EqD: equatorial diameter. Pollination strategy: A, anemophilous; Z, zoophilous.

\begin{tabular}{|c|c|c|c|c|c|c|c|c|c|}
\hline $\begin{array}{l}\text { FAMILY / Genus or species/ } \\
\text { PoLLEN TYPE }\end{array}$ & $\begin{array}{l}\text { Pollen } \\
\text { unit }\end{array}$ & Symmetry & Polarity & Size $[\mu m]$ & Aperture & Exine & Shape & $\begin{array}{l}\text { Pollination } \\
\text { strategy }\end{array}$ & Observations \\
\hline $\begin{array}{l}\text { EPHEDRACEAE Dumort. } \\
\text { Ephedra triandra Tul. } \\
\text { Type 01. EPHEDRA TRIANDRA } \\
\text { [Fig.4A] }\end{array}$ & monad & radiosymmetric & isopolar & $\begin{array}{l}\text { EqD: } 55(60) 62 ; \\
\text { PD: } 15(19) 25\end{array}$ & polyplicate & psilate & peroblate & A & \\
\hline $\begin{array}{l}\text { LAURACEAE Juss. } \\
\text { Ocotea Aubl. } \\
\text { Type 02. OcoteA [Fig.4B] }\end{array}$ & monad & radiosymmetric & apolar & $26(27) 29$ & inaperturate & echinate & spheroidal & Z & \\
\hline $\begin{array}{l}\text { AMARYLLIDACEAE J. St.-Hil. } \\
\text { Type 03. AMARYLLIDACEAE } \\
\text { [Fig.4C] }\end{array}$ & monad & heteropolar & bilateral & $\begin{array}{l}\text { Very variable. } \\
\text { Longer EqD: } \\
\text { 28(48)82; shorter } \\
\text { EqD: } 18(26) 39(\mathrm{PV})\end{array}$ & monosulcate & $\begin{array}{l}\text { reticulate, } \\
\text { mostly } \\
\text { homobrochate }\end{array}$ & elliptical (PV) & Z & \\
\hline $\begin{array}{l}\text { ARECACEAE Bercht. \& J. Presl } \\
\text { Butia odorata (Barb. Rodr.) } \\
\text { Noblick } \\
\text { Type 04. ButiA [Fig.4D] }\end{array}$ & monad & heteropolar & bilateral & $\begin{array}{c}\text { Longer EqD: } \\
\text { 52(63)79; shorter } \\
\text { EqD: } 23(32) 44(\mathrm{PV})\end{array}$ & monosulcate & perforate & $\begin{array}{l}\text { elliptical to } \\
\text { pyriform (PV) }\end{array}$ & Z & \\
\hline CYPERACEAE Juss. & monad & radiosymmetric & $\begin{array}{l}\text { isopolar or } \\
\text { heteropolar }\end{array}$ & $\begin{array}{l}\text { Very variable. } \\
\text { Length: } 33(42) 47 ; \\
\text { r width: } 28(33) 42 \\
(E q V)\end{array}$ & $\begin{array}{l}\text { inaperturate } \\
\text { or with } \\
\text { rudimentary } \\
\text { pores }\end{array}$ & $\begin{array}{l}\text { psilate to } \\
\text { scabrate }\end{array}$ & $\begin{array}{l}\text { spheroidal to } \\
\text { pyriform (EqV); } \\
\text { sub-circular (PV) }\end{array}$ & A & \\
\hline POACEAE Barnhart & & & & & & & & & \\
\hline Type 06. POACEAE [Fig.4F] & monad & radiosymmetric & heteropolar & $\begin{array}{c}\text { Very variable. } \\
19(36) 62\end{array}$ & monoporate & $\begin{array}{l}\text { psilate to } \\
\text { scabrate, rarely } \\
\text { granulate }\end{array}$ & spheroidal & A & $\begin{array}{l}\text { pores usually } \\
\text { with annulus } \\
\text { and sometimes } \\
\text { operculated }\end{array}$ \\
\hline $\begin{array}{l}\text { RANUNCULACEAE Juss. } \\
\text { Type 07. RANUNCULACEAE } \\
\text { [Fig.4G] }\end{array}$ & monad & radiosymmetric & isopolar & $\begin{array}{c}\text { EqD: } 22(25) 28 \text { PD: } \\
21(24) 28\end{array}$ & tricolporate & microechinate & $\begin{array}{l}\text { oblate spheroidal } \\
\text { (EqV); circular } \\
\text { (PV) }\end{array}$ & Z & \\
\hline $\begin{array}{l}\text { HALORAGACEAE R. Br. } \\
\text { Myriophyllum L. }\end{array}$ & & & & & & & & & \\
\hline $\begin{array}{l}\text { Type 08. MYRIOPHYLLUM } \\
\text { [Fig.4H] }\end{array}$ & monad & radiosymmetric & isopolar & EqD: $20(24) 28$ & $\begin{array}{l}\text { zonoporate } \\
\text { (4 pores) }\end{array}$ & $\begin{array}{l}\text { psilate to } \\
\text { scabrate }\end{array}$ & $\begin{array}{c}\text { suboblate to } \\
\text { oblate (EqV); } \\
\text { subcuadrangular } \\
\text { (PV) }\end{array}$ & A & $\begin{array}{l}\text { circular-elliptical } \\
\text { pores with } \\
\text { annulus }\end{array}$ \\
\hline $\begin{array}{l}\text { VITACEAE Juss. } \\
\text { Cissus L. }\end{array}$ & & & & & & & & & \\
\hline Type 09. Cissus [Fig.4I-J] & monad & radiosymmetric & isopolar & $\begin{array}{c}\text { EqD: } 29(33) 38 ; \mathrm{PD}: \\
50(57) 64\end{array}$ & tricolporate & $\begin{array}{c}\text { reticulate, } \\
\text { heterobrochate }\end{array}$ & $\begin{array}{l}\text { prolate }(\mathrm{EqV}) \\
\text { circular }(\mathrm{PV})\end{array}$ & Z & \\
\hline $\begin{array}{l}\text { OXALIDACEAE R. Br. } \\
\text { Oxalis L. }\end{array}$ & & & & & & - & & & \\
\hline Type 10. OxaLIS [Fig.4K] & monad & radiosymmetric & isopolar & $\begin{array}{c}\text { EqD: } 44(48) 53 ; \mathrm{PD}: \\
\text { 55(59)63 }\end{array}$ & tricolporate & $\begin{array}{c}\text { reticulate, } \\
\text { heterobrochate }\end{array}$ & $\begin{array}{c}\text { prolate to } \\
\text { subprolate (EqV); } \\
\text { subcircular (VP) }\end{array}$ & Z & \\
\hline $\begin{array}{l}\text { EUPHORBIACEAE Juss. } \\
\text { Sapium Jacq. }\end{array}$ & & & & & & & & & \\
\hline Type 11. SAPIUM [Fig.4L-M] & monad & radiosymmetric & isopolar & $\begin{array}{c}\text { EqD: } 34(38) 42 ; \mathrm{PD}: \\
51(64) 69\end{array}$ & tricolporate & microreticulate & $\begin{array}{l}\text { prolate to } \\
\text { perprolate (EqV); } \\
\text { circular (VP) }\end{array}$ & Z & endocingulum \\
\hline
\end{tabular}


Table 3. Cont.

\begin{tabular}{|c|c|c|c|c|c|c|c|c|c|}
\hline $\begin{array}{l}\text { FAMILY / Genus or species/ } \\
\text { PoLLEN TYPE }\end{array}$ & $\begin{array}{l}\text { Pollen } \\
\text { unit }\end{array}$ & Symmetry & Polarity & Size $[\mu m]$ & Aperture & Exine & Shape & $\begin{array}{l}\text { Pollination } \\
\text { strategy }\end{array}$ & Observations \\
\hline \multicolumn{10}{|l|}{ Sebastiania Spreng. } \\
\hline $\begin{array}{l}\text { Type 12. Sebastiania } \\
\text { [Fig. } 4 \mathrm{~N}-\mathrm{O}]\end{array}$ & monad & radiosymmetric & isopolar & $\begin{array}{c}\text { EqD: } 30(41) 49 ; \mathrm{PD}: \\
55(58) 66\end{array}$ & tricolporate & reticulate & $\begin{array}{l}\text { prolate }(\mathrm{EV}) \text {; } \\
\text { circular }(\mathrm{EqV})\end{array}$ & Z & \\
\hline \multicolumn{10}{|l|}{ PHYLLANTHACEAE Martinov } \\
\hline \multicolumn{10}{|l|}{$\begin{array}{l}\text { Phyllanthus } \\
\text { sellowianus (Klotzsch) Müll. Arg. }\end{array}$} \\
\hline $\begin{array}{l}\text { Type 13. Phyllanthus } \\
\text { SELLOWIANUS [Fig.4P] }\end{array}$ & monad & radiosymmetric & apolar & $16(17) 20$ & $\begin{array}{l}\text { pantoporate } \\
(12-15 \text { pores })\end{array}$ & clypeate & spheroidal & Z & \\
\hline \multicolumn{10}{|l|}{ SALICACEAE Mirb. } \\
\hline \multicolumn{10}{|l|}{ Salix chilensis Molina } \\
\hline $\begin{array}{l}\text { Type 14. SALIX CHILENSIS } \\
\text { [Fig.4Q-R] }\end{array}$ & monad & radiosymmetric & isopolar & $\begin{array}{c}\text { EqD: } 20(25) 32 ; \mathrm{PD}: \\
\text { 19(21)25 }\end{array}$ & tricolporate & $\begin{array}{c}\text { Reticulate, } \\
\text { heterobrochate }\end{array}$ & $\begin{array}{l}\text { spheroidal to } \\
\text { prolate (EqV); } \\
\text { circular (PV) }\end{array}$ & $\mathrm{Z}$ & \\
\hline \multicolumn{10}{|l|}{ FABACEAE Lindl. } \\
\hline \multicolumn{10}{|l|}{ Acacia Mill. } \\
\hline Type 15. AcACIA [Fig.4S] & polyad & & & $38(42) 45$ & $\begin{array}{l}\text { indistinguishable } \\
\text { pores }\end{array}$ & $\begin{array}{l}\text { psilate to slightly } \\
\text { scabrate }\end{array}$ & & Z & 16 pollen grains \\
\hline \multicolumn{10}{|l|}{$\begin{array}{l}\text { POLYGALACEAE Hoffmanns. } \\
\text { \& Link }\end{array}$} \\
\hline $\begin{array}{l}\text { Type 16. PolygalaceaE } \\
\text { [Fig. } 4 \text { T] }\end{array}$ & monad & radiosymmetric & isopolar & $\begin{array}{c}\text { EqD: } 16(17) 19 ; \mathrm{PD}: \\
21(25) 33\end{array}$ & $\begin{array}{l}\text { zonocolporate } \\
(10-12 \text { colpori) }\end{array}$ & psilate & $\begin{array}{l}\text { prolate (EqV); } \\
\text { circular to } \\
\text { multilobate (VP) }\end{array}$ & Z & endocingulum \\
\hline \multicolumn{10}{|l|}{ CANNABACEAE Martinov } \\
\hline \multicolumn{10}{|l|}{ Celtis L. } \\
\hline Type 17. Celtis [Fig.4U] & monad & radiosymmetric & isopolar & $\begin{array}{c}\text { EqD: } 25(29) 34 \text {; PD: } \\
\text { 16(17)18 }\end{array}$ & triporate & $\begin{array}{l}\text { psilate to } \\
\text { scabrate }\end{array}$ & $\begin{array}{l}\text { suboblate }(\mathrm{EqV}) \\
\text { circular }(\mathrm{PV})\end{array}$ & $\mathrm{A}-\mathrm{Z}$ & $\begin{array}{l}\text { pores with } \\
\text { annulus }\end{array}$ \\
\hline \multicolumn{10}{|l|}{ RHAMNACEAE Juss. } \\
\hline $\begin{array}{l}\text { Type 18. RHAMNACEAE } \\
\text { [Fig. } 4 \mathrm{~V} \text { ] }\end{array}$ & monad & radiosymmetric & isopolar & $\begin{array}{c}\text { EqD: } 17(20) 24 \text {; PD: } \\
\text { 17(21)25 }\end{array}$ & tricolporate & $\begin{array}{c}\text { vaguely } \\
\text { reticulate, } \\
\text { heterobrochate }\end{array}$ & $\begin{array}{c}\text { prolate } \\
\text { spheroidal (EqV); } \\
\text { subtriangular } \\
\text { (PV) }\end{array}$ & ; & \\
\hline \multicolumn{10}{|l|}{ MYRTACEAE Juss. } \\
\hline Type 19. MyrtaCeAe [Fig.5A] & monad & radiosymmetric & isopolar & $\begin{array}{l}\text { EqD: } 17(24) 27 ; \text { PD: } \\
\text { 11(12)15 P }\end{array}$ & syntricolporate & $\begin{array}{l}\text { psilate to slightly } \\
\text { scabrate }\end{array}$ & $\begin{array}{l}\text { peroblate }(\mathrm{EqV}) \\
\text { triangular to } \\
\text { subtriangular } \\
(\mathrm{PV})\end{array}$ & Z & $\begin{array}{c}\text { rarely } \\
\text { syntetracolporate }\end{array}$ \\
\hline \multirow{2}{*}{\multicolumn{10}{|c|}{$\begin{array}{l}\text { THYMELAEACEAE Juss. } \\
\text { Daphnopsis racemosa Griseb. }\end{array}$}} \\
\hline & & & & & & & & & \\
\hline $\begin{array}{l}\text { Type 20. DAPHNOPSIS } \\
\text { RACEMOSA [Fig.5B-C] }\end{array}$ & monad & radiosymmetric & apolar & 29(35)39 & $\begin{array}{l}\text { pantoporate } \\
(12-15 \text { pores })\end{array}$ & croton pattern & spheroidal & Z & \\
\hline \multicolumn{10}{|l|}{ ANACARDIACEAE R. Br. } \\
\hline $\begin{array}{l}\text { Type 21. Lithraea/Schinus } \\
\text { [Fig.5D-E] }\end{array}$ & monad & radiosymmetric & isopolar & $\begin{array}{c}\text { EqD: } 23(27) 31 \text {; PD: } \\
27(40) 45\end{array}$ & tricolporate & $\begin{array}{l}\text { striate to } \\
\text { reticulate }\end{array}$ & $\begin{array}{c}\text { prolate to } \\
\text { subprolate (EqV); } \\
\text { circular (VP) }\end{array}$ & Z & \\
\hline $\begin{array}{l}\text { SAPINDACEAE Juss. } \\
\text { Allophylus edulis (A. St.-Hil., A. } \\
\text { Juss. \& Cambess.) Hieron. ex } \\
\text { Niederl. }\end{array}$ & & & & & & & & & \\
\hline
\end{tabular}


Table 3. Cont.

\begin{tabular}{|c|c|c|c|c|c|c|c|c|c|}
\hline $\begin{array}{l}\text { FAMILY / Genus or species/ } \\
\text { PoLLEN TYPE }\end{array}$ & $\begin{array}{l}\text { Pollen } \\
\text { unit }\end{array}$ & Symmetry & Polarity & Size $[\boldsymbol{\mu m}]$ & Aperture & Exine & Shape & $\begin{array}{l}\text { Pollination } \\
\text { strategy }\end{array}$ & Observations \\
\hline $\begin{array}{l}\text { Type 22. AlLOPHYLUS EDULIS } \\
\text { [Fig.5F] }\end{array}$ & monad & radiosymmetric & isopolar & $\begin{array}{c}\text { EqD: } 27(30) 32 ; \mathrm{PD}: \\
\text { 18(19)21 }\end{array}$ & $\begin{array}{l}\text { zonoporate } \\
\text { (4 pores) }\end{array}$ & microreticulate & $\begin{array}{l}\text { oblate }(\mathrm{EqV}) \text {; } \\
\text { quadrangular } \\
\qquad(\mathrm{PV})\end{array}$ & Z & rarely triporate \\
\hline $\begin{array}{l}\text { Dodonaea viscosa Jacq. } \\
\text { Type 23. DodonAEA viscosA } \\
\text { [Fig.5G-H] }\end{array}$ & monad & radiosymmetric & isopolar & $\begin{array}{c}\text { EqD: 25(28)33; PD: } \\
\text { 24(27)31 }\end{array}$ & tricolporate & verrucate & $\begin{array}{l}\text { prolate } \\
\text { spheroidal (EqV); } \\
\text { subcircular (PV) }\end{array}$ & Z & $\begin{array}{l}\text { rarely } \\
\text { tetracolporate }\end{array}$ \\
\hline \multicolumn{10}{|l|}{ LORANTHACEAE Juss. } \\
\hline $\begin{array}{l}\text { Tripodanthus acutifolius (Ruiz \& } \\
\text { Pav.) Tiegh } \\
\text { Type 24. TRIPODANTHUS } \\
\text { ACUTIFOLIUS [Fig.5I] }\end{array}$ & monad & radiosymmetric & isopolar & $\begin{array}{c}\text { EqD: } 23(25) 28 ; \mathrm{PD}: \\
\text { 10(12)15 }\end{array}$ & syntricolporate & $\begin{array}{l}\text { psilate, baculate } \\
\text { at mesocolpium }\end{array}$ & $\begin{array}{l}\text { peroblate }(\mathrm{EqV}) \\
\text { subtriangular } \\
(\mathrm{PV})\end{array}$ & Z & $\begin{array}{c}\text { rarely } \\
\text { syntetracolporate }\end{array}$ \\
\hline \multicolumn{10}{|l|}{$\begin{array}{l}\text { AMARANTHACEAE Juss. } \\
\text { Amaronthoideae }\end{array}$} \\
\hline $\begin{array}{l}\text { Type } 25 . \text { AltERnANTHERA- } \\
\text { TYPE [Fig.5J] }\end{array}$ & monad & radiosymmetric & apolar & $15(19) 24$ & $\begin{array}{l}\text { pantoporate } \\
\text { (12-16 pores) }\end{array}$ & reticulate & $\begin{array}{l}\text { spheroidal- } \\
\text { polyhedral }\end{array}$ & Z & \\
\hline $\begin{array}{l}\text { Type 26. GOMPHRENA-TYPE } \\
\text { [Fig.5K-L] }\end{array}$ & monad & radiosymmetric & apolar & $15(19) 23$ & $\begin{array}{l}\text { pantoporate } \\
\text { ( }>20 \text { pores })\end{array}$ & reticulate & spheroidal & Z & \\
\hline $\begin{array}{l}\text { Chenopodioideae } \\
\text { Type 27. CHENOPODIOIDEAE } \\
\text { [Fig.5M] }\end{array}$ & monad & radiosymmetric & apolar & $20(24) 26$ & $\begin{array}{l}\text { pantoporate } \\
(>35 \text { pores })\end{array}$ & psilate & spheroidal & A & $\begin{array}{l}\text { pores with thin } \\
\text { annulus }\end{array}$ \\
\hline $\begin{array}{l}\text { CARYOPHYLLACEAE Juss. } \\
\text { Type 28. CARYOPHYLLACEAE } \\
\text { [Fig.5N-O] }\end{array}$ & monad & radiosymmetric & apolar & $26(32) 36$ & $\begin{array}{l}\text { pantoporate } \\
\text { ( } 15 \text { pores })\end{array}$ & $\begin{array}{l}\text { psilate and } \\
\text { slightly perforate }\end{array}$ & spheroidal & Z & $\begin{array}{l}\text { pores with } \\
\text { annulus }\end{array}$ \\
\hline $\begin{array}{l}\text { POLYGONACEAE Juss. } \\
\text { Polygonum L. } \\
\text { Type } 29 . \\
\text { POLYGONUM [Fig.5P-Q] }\end{array}$ & monad & radiosymmetric & apolar & $40(54) 64$ & $\begin{array}{c}\text { pantoporate } \\
\text { ( } \sim 10 \text { pores })\end{array}$ & reticulate & spheroidal & $\mathrm{Z}$ & \\
\hline \multicolumn{10}{|l|}{$\begin{array}{l}\text { PRIMULACEAE Batsch ex } \\
\text { Borkh. }\end{array}$} \\
\hline $\begin{array}{l}\text { Myrsine L. } \\
\text { Type 30. Myrsine [Fig.5R] }\end{array}$ & monad & radiosymmetric & isopolar & $\begin{array}{c}\text { EqD: } 16(20) 23 ; \mathrm{PD}: \\
19(22) 25\end{array}$ & $\begin{array}{l}\text { zonocolporate } \\
\text { ( } 4 \text { colpori) }\end{array}$ & psilate & $\begin{array}{c}\text { prolate } \\
\text { spheroidal } \\
\text { (EqV); circular to } \\
\text { tetrangular (PV) }\end{array}$ & $A-Z$ & $\begin{array}{l}\text { rarely } 3-5 \text { colpori; } \\
\text { pore mostly } \\
\text { indistinguishable }\end{array}$ \\
\hline $\begin{array}{l}\text { RUBIACEAE Juss. } \\
\text { Cephalanthus glabratus (Spreng.) }\end{array}$ & & & & & & & & & \\
\hline $\begin{array}{l}\text { Type 31. CEPHALANTHUS } \\
\text { GLABRATUS [Fig.5S] }\end{array}$ & monad & radiosymmetric & isopolar & $\begin{array}{c}\text { EqD: } 18(23) 28 ; P D: \\
20(22) 23\end{array}$ & tricolporate & $\begin{array}{l}\text { Reticulate, } \\
\text { homobrochate }\end{array}$ & $\begin{array}{l}\text { sub-spheroidal } \\
\text { (EqV); circular to } \\
\text { sub-triangular } \\
\text { (PV) }\end{array}$ & $\mathrm{Z}$ & $\begin{array}{l}\text { pores with } \\
\text { annulus }\end{array}$ \\
\hline \multicolumn{10}{|l|}{ PLANTAGINACEAE Juss. } \\
\hline Type 32. Plantago [Fig.5T] & monad & radiosymmetric & apolar & $28(39) 32$ & $\begin{array}{l}\text { pantoporate } \\
(\sim 7 \text { pores })\end{array}$ & verrucate & spheroidal & $\mathrm{A}$ & $\begin{array}{l}\text { faint irregular } \\
\text { pores }\end{array}$ \\
\hline \multicolumn{10}{|l|}{ ASTERACEAE Bercht. \& J. Presl } \\
\hline $\begin{array}{l}\text { Type 33. AsteraceAe SUbF. } \\
\text { Asteroideae [Fig.5U-V] }\end{array}$ & monad & radiosymmetric & isopolar & $\begin{array}{c}\text { EqD: } 15(28) 40 ; \text { PD: } \\
\text { 20(33) } 43\end{array}$ & tricolporate & $\begin{array}{c}\text { echinate or } \\
\text { subechinolophate }\end{array}$ & $\begin{array}{c}\text { prolate } \\
\text { spheroidal to } \\
\text { subprolate (EqV); } \\
\text { circular to sub- } \\
\text { triangular (PV) }\end{array}$ & ; & \\
\hline \multicolumn{10}{|l|}{$\begin{array}{l}\text { MENYANTHACEAE Dumort. } \\
\text { Nymphoides indica (L.) Kuntze }\end{array}$} \\
\hline $\begin{array}{l}\text { Type 34. NyMPHOIDES INDICA } \\
\text { [Fig.5W] }\end{array}$ & monad & radiosymmetric & isopolar & $\begin{array}{c}\text { EqD: } 39(41) 43 ; \mathrm{PD}: \\
22(24) 28\end{array}$ & syntricolporate & microbaculate & $\begin{array}{l}\text { oblate }(\mathrm{EqV}) \\
\text { triangular }(\mathrm{PV})\end{array}$ & Z & \\
\hline APIACEAE Lindl. & & & & & & & & & \\
\hline $\begin{array}{l}\text { Eryngium L. } \\
\text { Type 35. ERYNGIUM [Fig.5X-Y] }\end{array}$ & monad & radiosymmetric & isopolar & $\begin{array}{l}\text { EqD: } 14(18) 23 ; \\
\text { PD: } 32(42) 48\end{array}$ & tricolporate & psilate & $\begin{array}{c}\text { perprolate } \\
\text { (EqV); circular } \\
\text { (PV) }\end{array}$ & Z & \\
\hline
\end{tabular}




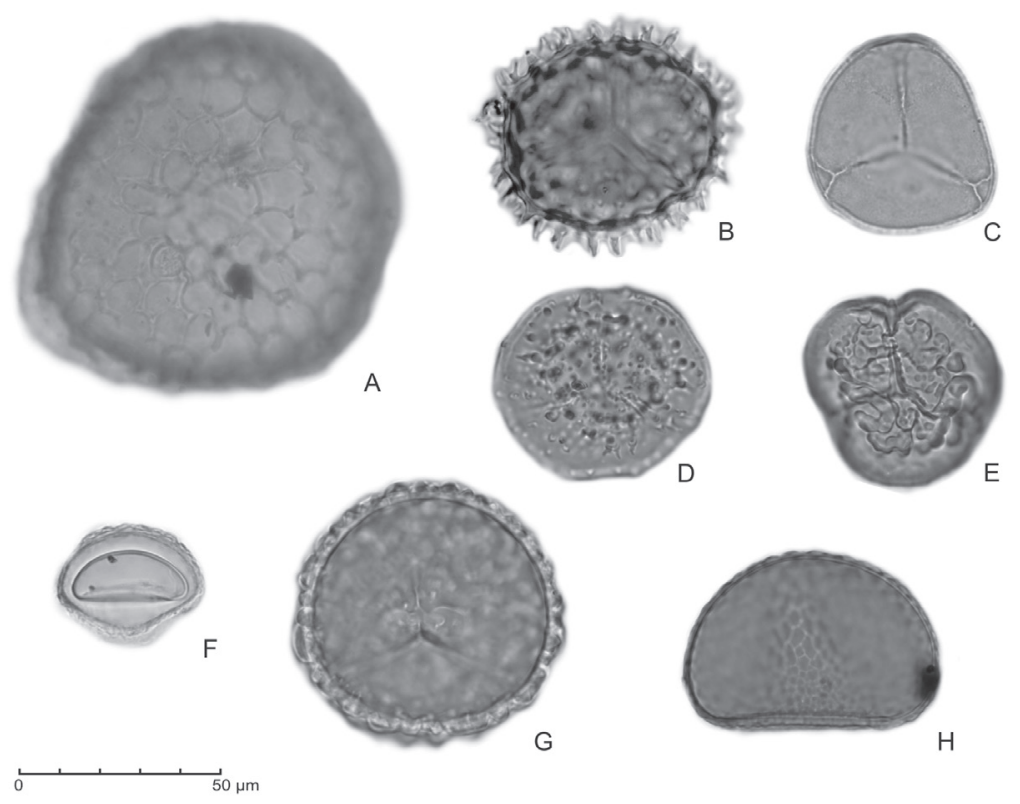

Figure 3. Photomicrographs of spores from the campos region of Uruguay. A - Type 01 RICCIACEAE; B - Type 02 ANTHOCEROS; C - Type 03 Phaeoceros bulbiculosus; D - Type 04 Phaeoceros laevis; E - Type 05 Phaeoceros tenuis; F - Type 06 Isoetes; G - Type 07 Ophioglossum; H - Type 08 Polypodiaceae.

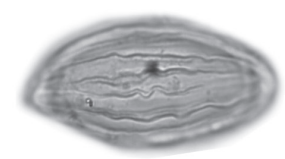

A

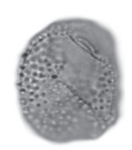

B

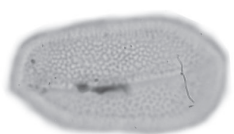

C

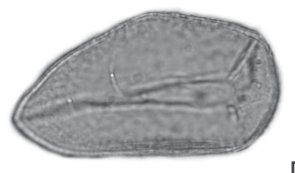

$\mathrm{D}$
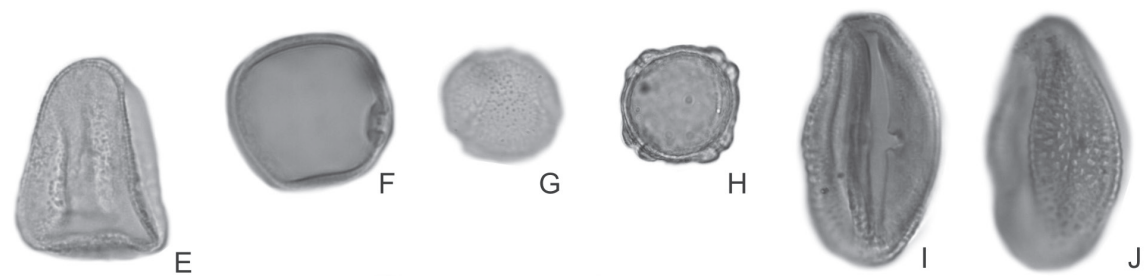

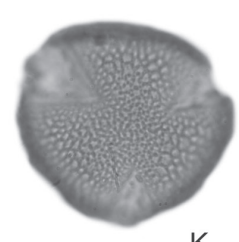

$\mathrm{K}$

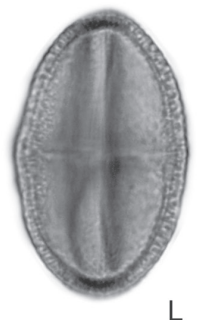

$\mathrm{L}$

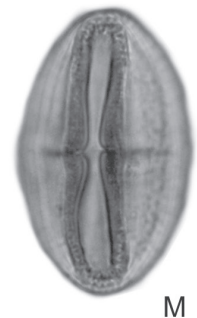

$\mathrm{M}$

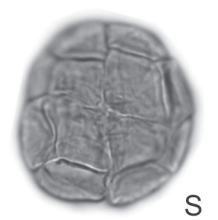

T
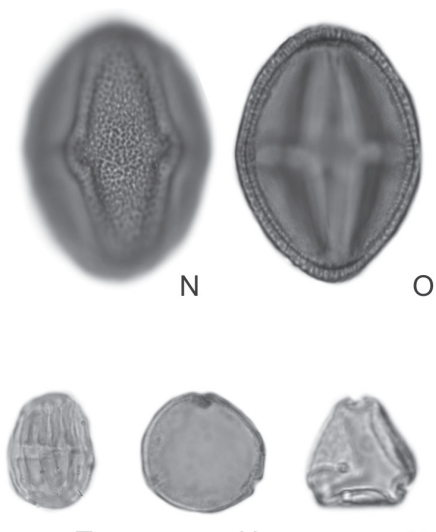

$\mathrm{O}$

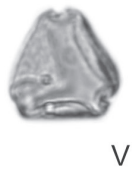

Figure 4. Photomicrographs of pollen from the campos region of Uruguay. A - Type 01 EpHEDRA TRIANDRA; B - Type 02 OcoteA; C - Type 03 AmaryllidaceaE; D - Type 04 Butia; E - Type 05 Cyperaceae; F - Type 06 Poaceae; G - Type 07 RanunculaceaE; H - Type 08 Myriophyllum; I-J - Type 09 Cissus; K - Type 10 Oxalis; L-M - Type 11 Sapium; N-O - Type 12 Sebastiania; P Type 13 Phyllanthus sellowianus; Q-R Type 14 Salix chilensis; S - Type 15 Acacia; T - Type 16 Polygalaceae; U - Type 17 Celtis; V - Type 18 RhamnaceaE. 


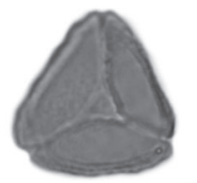

A

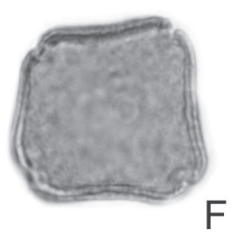

$\mathrm{F}$

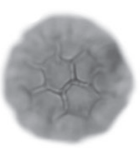

K
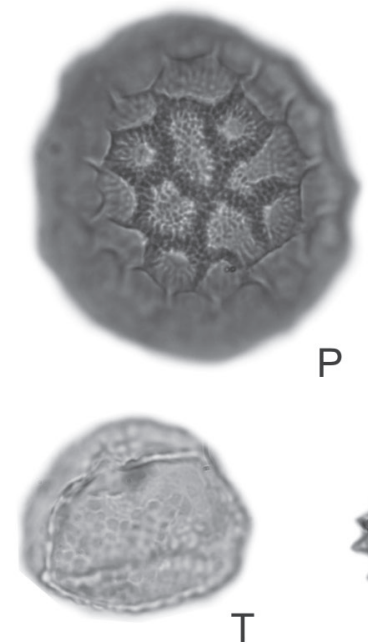

$\mathrm{T}$

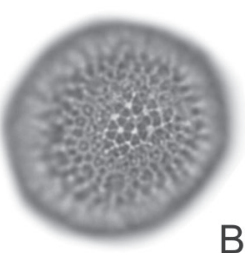

B

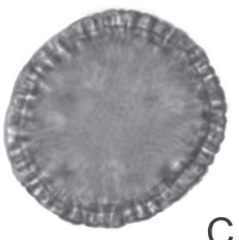

C
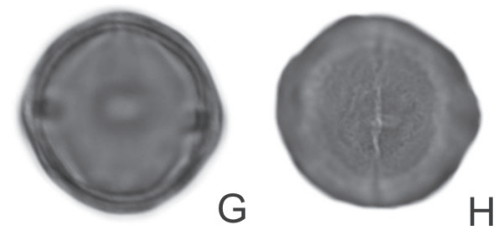

$\mathrm{H}$
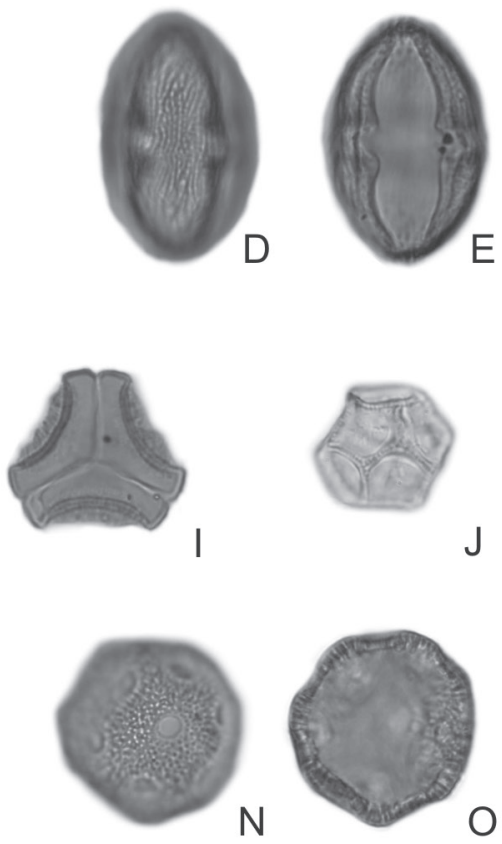

$\mathrm{N}$

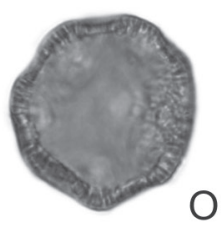

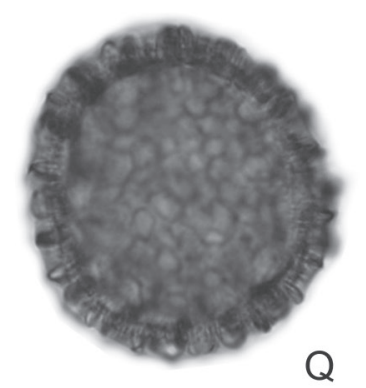
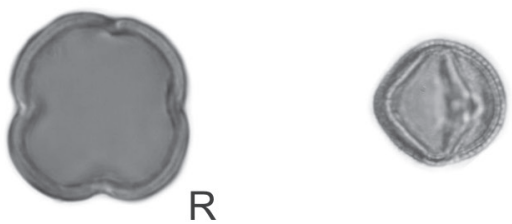

S
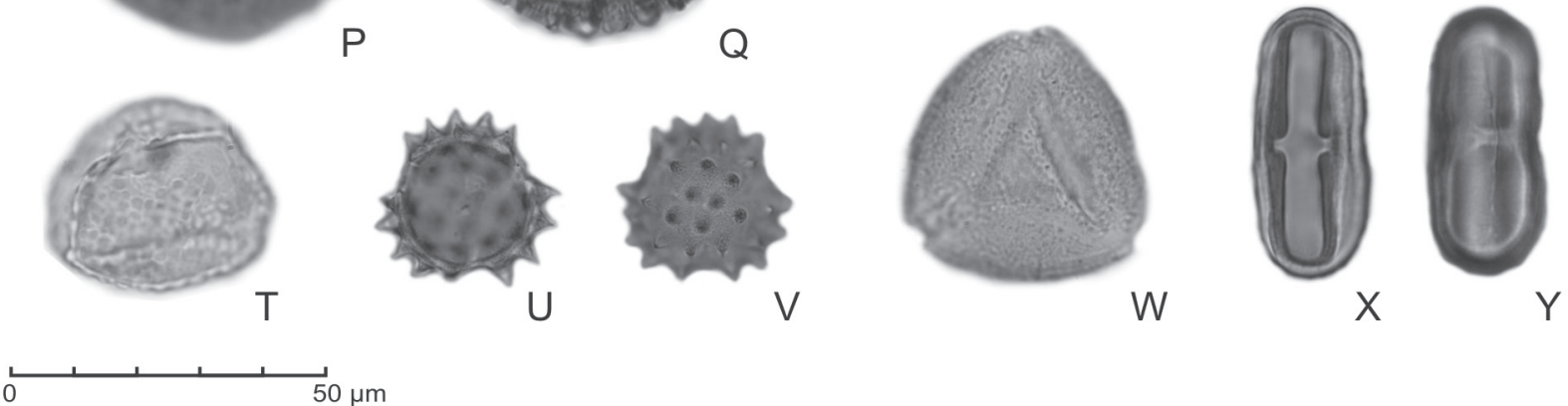

W

Y

Figure 5. Photomicrographs of pollen from the campos region of Uruguay. A - Type 19 MyrTACEAE; B-C - Type 20 DAPHNOPSIS RACemosa; D-E - Type 21 Lithraea/Schinus; F - Type 22 Allophylus edulis; G-H - Type 23 DodonAeA VIScosa; I - Type 24 Tripodanthus acutifolius; J - Type 25 Alternanthera-Type; K-L - Type 26 GomphrenA-Type; M - Type 27 Chenopodioideae; N-O - Type 28 Caryophyllaceae; P-Q - Type 29 Polygonum; R - Type 30 Myrsine; S - Type 31 Cephalanthus glabratus; T Type 32 Plantago; U-V - Type 33 Asteraceae subf. Asteroideae; W - Type 34 Nymphoides indica; X-Y - Type 35 Eryngium.

family in natural grasslands, mainly represented by genera such as Baccharis and Eupatorium (Tab. 1). CYPERACEAE is over-represented in some samples where small local communities related to higher substrate water availability are observed (samples 5, 42, 53, 54, 57; Fig. 2) (Mourelle \& Prieto 2012). Apiaceae is also well represented in pollen assemblages, principally due to Eryngium sp. (ERYNGIUM, Fig. 5X-Y), which is commonly recorded in grasslands.

Other graminoid herbs are also frequent in the pollen assemblages. For instance, Amaranthaceae family is represented by pollen grains of the genera Alternanthera spp. (Alternanthera-type, Fig. 5J), Gomphrena spp. (GomphrenA-TYPe, Fig. 5K-L) and Chenopodium spp. (Chenopodioideae, Fig. 5M). Monocotyledoneae corresponds to various families such as Juncaceae, Amaryllidaceae (AmARYLlidACEAE, Fig. 4C) and Iridaceae. RANUNCULACEAE (Fig. 4G) mainly represents the common species Anemone decapetala, and CARYOPHYLlaCEAE (Fig. $5 \mathrm{~N}-\mathrm{O})$ mainly Cerastium spp. and Silene spp. Other taxa widely dispersed in the campos are represented by OxALIS (Fig. 4K), Plantago (Fig. 5T) and Polygalaceae (Fig. 4T). Bryophytes are dominated by PHAEOcERos (Fig. 3C-E) 


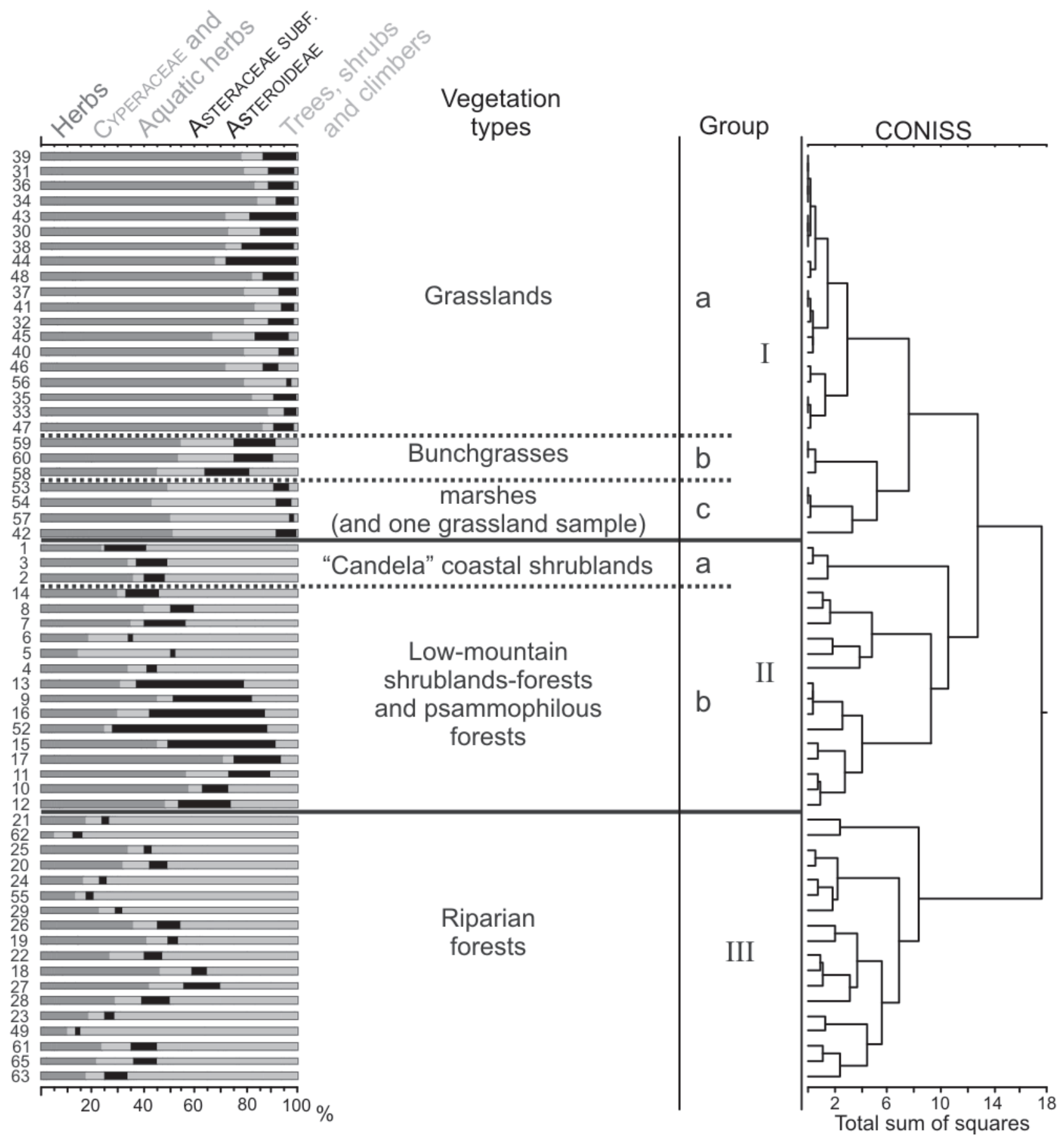

Figure 6. Summary percentage pollen diagram of the 62 surface soil samples ordered according to the Cluster Analysis unconstrained results.

and Anthoceros (Fig. 3B), frequent in South American moist soils (Villarreal et al. 2010); and pteridophytes by IsOETES (Fig. 3F) and OpHIOGLOSSUM (Fig. 3G) spores from small plants that grow between grasses and graminoids. RICCIACEAE (Fig. 3A) is present in some samples and could represent muddy areas.

In grassland surface samples shrubby and arboreal pollen is present in traces, mainly represented by mesophilous taxa (Myrtaceae; Myrsine, Fig. 5-R and Celtis, Fig. 4U). Blepharocalyx salicifolius (Myrtaceae) and Myrsine laetevirens trees reach up to 10-15 m height and constitute the forest canopy, over which Celtis iguanaea climbs. As a result, pollen of these mesophilous taxa, besides falling gravitationally into the forest, can be more easily dispersed into grasslands than pollen from hydrophilous taxa, which only reach up to $4 \mathrm{~m}$ height and grow restricted to water body margins 
(e.g. Phyllanthus sellowianus and Cephalanthus glabratus). In addition, the frequent presence of CELTIS and MYRSINE in grassland samples could be related to wind dispersal (Otegui \& Cocucci 1999; Gassmann \& Pérez 2006; Torretta \& Basilio 2009).

Uniform morphology of POACEAE pollen presents problems in the reconstruction of past grassland ecosystems and, therefore, many efforts have been made in order to elaborate a method to differentiate grassland ecosystems based on measurable POACEAE pollen parameters. Jan et al. (2015) observed that neither the scores of C3/C4 Poaceae species nor those diploid/polyploid species are clearly separable, despite there are clear trends showing that pollen sizes are larger in $C 4$ as compared with $C 3$ species, buy also polyploids are larger than diploid species. Grasslands dominated by $\mathrm{C} 3$ and $\mathrm{C} 4$ Poaceae species from different regions and habitats could then be separated by studying the pattern of trends in their pollen size (Jan et al. 2015). In this regard, Schüler \& Behling (2011) used POACEAE pollen size as a tool for distinguishing past grasslands in South America, demonstrating that a distinction between grassland ecosystems is possible based on measurable POACEAE pollen grain parameters. They observed that the RPG POACEAE pollen was considerably smaller compared to the one from other grassland regions, but they did not find significant differences between POACEAE pollen from the campos and the pampas regions. In particular, for many taxa from different genus or tribes present in the campos region, only the extreme forms are readily separable, but there is a continuum of forms blurring the distinction between the extremes (Radaeski 2014). A false separation of forms is statistically more misleading than a lumping of types, although this involves losing ecological information for paleoecological reconstructions. In consequence, we still suggest considering POACEAE pollen grains at the family level in combination with minor taxa of grasslands. This also applies to Asteraceae SUbF. Asteroideae which, despite differences between their pollen grains are clearer (e.g. Punt \& Hoen 2009; Cancelli et al. 2010), the difficulty classifying all AsteraceAe SUBF. Asteroideae pollen present in modern and fossil assemblages might generate misleading paleoecological interpretations. Therefore, we propose considering them at the subfamily level. Therefore, the limited taxonomic resolution of the most abundant pollen in grassland samples (PoACEAE, Cyperaceae and Asteraceae sUbF. Asteroideae) makes it impossible to differentiate samples from northern and southern campos at a regional scale since this division is based on different Poaceae genera (Mourelle \& Prieto 2012). However, some divisions at a local scale are possible (see below).

The presence of shrubby and arboreal pollen in the campos differentiates their pollen assemblages from those of the pampas where these taxa are absent, taken into account that forest and shrubland hardly develop in the pampas region. Samples belonging to grasslands where palm individuals grow could be differentiated from the others by the presence of BUTIA pollen (Fig. 4D). However, not all surface samples from grasslands where palm individuals develop exhibited BuTiA pollen. This absence prevents us to interpret this as a lack of palms in the vegetation (Mourelle \& Prieto 2012).

\section{Marshes and bunchgrasses}

Pollen assemblages of brackish and freshwater marshes are co-dominated by PoAceae and Cyperaceae. Most POACEAE pollen comes from the regional grasslands, while CYPERACEAE comes from plants from soil depressions where water accumulates, promoting the local growth of species such as Schoenoplectus californicus and Scirpus giganteus (Rodríguez-Gallego et al. 2012) (Fig. 2; Tab. 1 ). The absence of Juncus ACUTUS in brackish marshes surface samples does not allow the differentiation between them and the freshwater marshes, and highlights the importance of identifying the silent taxa in order not to misinterpret the fossil pollen spectra. Furthermore, on the Uruguayan coast there are not salt marshes dominated by Chenopodioideae, as observed in other South American areas (Isacch et al. 2006) where CHENOPODIOIDEAE pollen often reaches a strong local representation (up to 80\%) in Bahia Samborombón (Vilanova \& Prieto 2012) and Laguna Mar Chiquita (Stutz \& Prieto 2003). This fact must be taken into account when interpreting coastal fossil sequences, as undertaken in ASG, where the development of salt marshes dominated by Chenopodioideae during the mid Holocene was inferred (Mourelle et al. 2015a). Bunchgrasses are dominated by Poaceae species that develop together with some Cyperaceae in soils with high substrate water availability, and hence POACEAE and CYPERACEAE also codominate their pollen assemblages (Fig. 2; Tab. 1). Relatively dense Eryngium pandanifolium clumps, locally known as "caraguatales", are associated to bunchgrasses and are also represented in their pollen spectra (ERYNGIUM). In addition to grasslands, both marshes and bunchgrasses are observed in open environments, which favored the deposition of woody pollen dispersed from the canopy of the neighboring riparian forests (Fig. 2).

\section{Forests}

The different forest types that develop in the campos region of Uruguay (Tab. 1) share most of their species, and are mainly composed of mesophilous trees and shrubs belonging to the families Myrtaceae (Blepharocalyx salicifolius, Myrceugenia glaucescens, Myrcianthes cisplatensis), Rhamnaceae (Scutia buxifolia) and Primulaceae (Myrsine coriacea, $M$. ferruginea), which are represented in the pollen samples by MYRTACEAE, RHAMNACEAE and MYRSINE. Other pollen types from mesophilous trees are ALLOPHYlus EDUlis (Fig. 5F), Lithraea/Schinus (Fig. 5D-E) and 
SAPIUM (Fig. 4L-M). All of these taxa constitute the core zone of the forests, and some of them reach the canopy on which top other species such as Celtis iguanaea (CELTIS) and Tripodanthus acutifolius (TRIPODANTHUS ACUTIFOlIUS, Fig. 5I) climb. On the outer zone of the forests other species, such as Daphnopsis racemosa (DAPHNOPSIS RACEMOSA, Fig. 5B-C), Acacia bonariensis (ACACIA, Fig. 4S) and Cissus palmata (CIssus, Fig. 4I-J) develop. The understorey supports many herbs, bryophytes and pteridophytes, represented in the pollen spectra by Poaceae, OXalis, Caryophyllaceae, Phaeoceros, Isoetes and Polypodiaceae (Fig. 3H). The fact that all these taxa are shared by different forest types developed in the campos region not only makes these pollen grains and spores characterize and define them in the palynological spectra but it also means that they are not useful to differentiate between forests.

For palynologists interpreting pollen diagrams is important to understand which groups of taxa are likely to be silent in the pollen record. For instance, pollen from the characteristic species of the psammophilous forest vegetation (Sideroxylon obtusifolium, Rollinia maritima and Varronia curassavica) were absent in the pollen assemblages (Fig. 2; Tab. 1). In consequence, such silent taxa prevent the psammophilous forest to be clearly identified from other forests in the pollen assemblages, mainly from low-mountain shrublands-forests (Fig. 6). However, it is important to note that pollen spectra from psammophilous forest have higher values of EPHEDRA TRIANDRA (Fig. 4A) and RHAMNACEAE, and minor of AsteraceAe SUbF. AsteroideAe, related to the abundance of their parental plants on each vegetation type (Figs. 2, 6; Tab. 1).

In contrast, some different vegetation types can be identified and statistically separated from others by a few key indicator taxa (e.g. Bush 1991; Rodgers III \& Horn 1996; Ortuño et al. 2011). Pollen samples from riparian forests are dominated by woody taxa, represented by the mesophilous taxa described above, but also by hydrophilous ones that develop exclusively in these forests (Tab. 1), represented in pollen spectra by SEBASTIANIA (Fig. 4N-O), SALIX CHILENSIS (Fig. 4Q-R), PHYLLANTHUS SELlOWIANUS (Fig. 4P) and Cephalanthus Glabratus (Fig. 5S). These pollen types are riparian forest key indicator taxa. Aquatic herbs such as Myriophyllum (Fig. 4H), Polygonum (5P-Q) and NympHoides INDicA (Fig. 5W) reflect plants that develop in the margin of the water bodies and in the floodplains. In addition, riparian forests understorey is more humid than in the other forest types, and that favored the development of Isoetes (ISOETES) and many pteridophytes of genera such as Campyloneurum, Pleopeltis and Microgramma (Polypodiaceae).

In riparian forest samples, some pollen types are markedly over-represented (e.g. MyrtaceAe, Myrsine, Sebastiania), while others are under-represented (OCOTEA, Fig. 4B) or absent (ERYTHRINA CRISTA-GALLI), even though these plants are frequent in the vegetation.
OcoтEA pollen grains are very fragile (Behling \& Negrelle 2006) and Erythrina crista-galli is pollinated by birds and so their flowers have stamens hidden deep in the corolla tube (Hidalgo 2008), two facts that make that these grains are not usually found in pollen assemblages. However, this does not generate problems concerning the correct identification of riparian forest pollen assemblages, both qualitatively (Fig. 2) and quantitatively (Fig. 6), principally due to the presence of the key indicator pollen types from hydrophilous taxa that grow exclusively in these forests.

Therefore, when we analyze the pollen spectra of a fossil sequence we must take into account that the presence of key pollen types from woody hydrophilous taxa indicate the development of riparian forests, whereas the presence of pollen types from mesophilous taxa do not allow us to distinguish what type of forest these pollen grains might be coming from.

\section{Shrublands}

There exists a considerable difference between shrubland and forest soils. Shrublands develop in soils with coarse sand, low humidity and water availability for plants, as well as low organic matter and nitrogen levels (Bartesaghi 2007). These conditions hinder the development of many taxa, mainly trees, bryophytes and pteridophytes.

DodonAEA VISCOSA (Fig. 5G-H) dominates the pollen spectra and vegetation from "Candela" coastal shrublands (Fig. 6; Tab. 1). Asteraceae subf. Asteroideae pollen is also well represented, mainly due to shrubs such as Baccharis spp. and Eupatorium buniifolium, and herbs such as Senecio spp.

\section{Woody pollen dispersal}

Woody taxa from riparian forests have mostly zoophilous pollination (Tab. 1) and so they produce low quantities of pollen grains poorly dispersed. As a consequence, most of the woody pollen grains produced by riparian forests drop directly into the ground and have strong local effect (Figs. $2,7)$. Inside the riparian forests, woody pollen represents ca. $70 \%$, while at distances $>250 \mathrm{~m}$ woody pollen does not reach up to $2 \%$ of the pollen assemblage, represented by mesophilous taxa (Myrtaceae, Myrsine and Celtis). Blepharocalyx salicifolius (Myrtaceae), Myrsine laetevirens and Celtis iguanaea reach riparian forest canopy and so some of their pollen grains can be dispersed away to grasslands. This could also be related to CELTIS and MYRSINE wind dispersal (Otegui \& Cocucci 1999; Gassmann \& Pérez 2006; Torretta \& Basilio 2009).

Pollination mechanisms will predictably bias the fossil record: pollen grains arriving to lake sediments are most likely to be from anemophilous taxa or from zoophilous ones exhibiting "messy" pollination syndromes, in the sense that excess pollen is spilled into the air, for example, due 


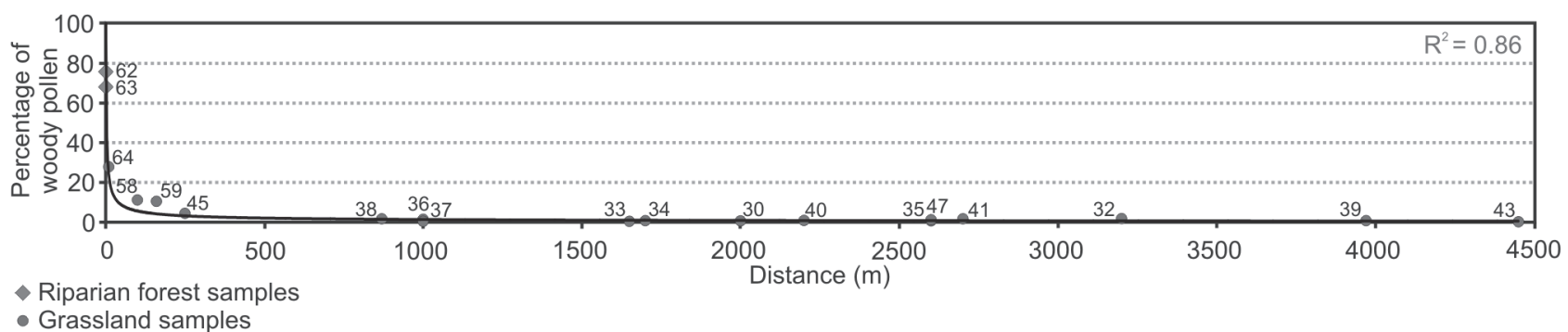

Figure 7. Woody pollen dispersal curve from riparian forests to grasslands. Two riparian forest samples were considered as pollen sources (samples 62 and 63). Numbers correspond to surface samples (see Fig. 1).

to vibrations from wings of a visiting bee (Bush \& Rivera 2001). Our results reflect that just very small amounts (or even traces) of key pollen types from hydrophilous taxa found in the fossil spectra from sequences taken in open environments (such as lagoons or marshes) indicate the development of riparian forests in the surroundings. However, further detailed investigations are required to obtain more robust results.

\section{Application to interpret Holocene sequences from the campos region}

Paleoecological investigations based on pollen and spores content in fossil sediments from campos region (ASG and PB-IM, Fig. 8) brought information about environmental and climatic changes impact in vegetation during the last ca. 8000 cal. yr BP. (1) Palynomorph analyses carried on ASG sequence from the marsh in the lower reach of Arroyo Solís Grande ( $34^{\circ} 45^{\prime} 35^{\prime} \mathrm{S}$ - $55^{\circ} 25^{\prime} 55^{\prime \prime} \mathrm{W}$; Figs. 1,8 ) allowed the reconstruction of vegetation history in the Río de la Plata northeastern coast (Uruguay) during the mid and late Holocene in relation to the relative sea-level fluctuations and the geomorphologic evolution (Mourelle et al. 2015a; Prieto et al. 2016). (2) Palynomorph analyses carried on PB-IM sequence from the wetland Paso Barranca-India

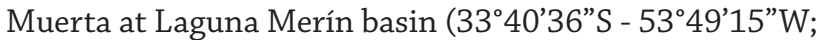
Figs. 1, 8) allowed the reconstruction of vegetation history during the late Holocene in relation to edaphic and climatic conditions (Mourelle et al. 2015b). Despite fossil pollen assemblages from campos region showed considerable pollen and spores diversity, for which it is indispensable to have a good palynomorph morphology knowledge, many taxa could only be identified at genus or family level, causing a loss of ecological information that had to be taken into account while reconstructing paleovegetation. However, the good relationship between modern pollen and spores assemblages and the principal vegetation types of the campos region of Uruguay provided useful data for the interpretation of Holocene pollen sequences from this area.

The dominance of POACEAE in the fossil pollen spectra makes it imperative to analyze the other variables in great detail, paying special attention to the pollen types representing small herbs and woody taxa. The knowledge of the pollination strategies of parental plants allows the evaluation of the significance of their pollen grains in the fossil spectra. Then, small changes in their proportions in fossil assemblages have been really relevant for making accurate paleoecological interpretations.

The pollen types that dominate the pollen assemblages of the two Holocene sequences studied (ASG and PB-IM, Fig. 8) represent anemophilous plants (POACEAE accompanied by Cyperaceae) associated to zoophilous ones (Asteraceae Subf. Asteroideae, Monocotyledoneae, Apiaceae and pollen from small herbs) (Fig. 2). These pollen assemblages represent the regional dominance of grasslands in this region during mid and late Holocene, with characteristics similar to the current ones.

Despite PoACEAE high proportions in ASG sequence are related to the dominance of grasslands all over the region, their grains may also have a local origin. Its co-dominance with Chenopodioideae and the geomorphological characteristics of the coastal area revealed the development of salt marshes around an estuary during the sea-level rise and highstand (between ca. 8000 and 2900 cal. yr BP). In consequence, POACEAE pollen probably also corresponds to Spartina sp., a dominant taxa in these environments. This scenario is comparable with the salt marsh vegetation that currently occurs in some coastal areas of the pampas (Mourelle et al. 2015a).

Amaranthoideae (Gomphrena-type and ALtERNANTHERA-TYPE) and RANUNCULACEAE represent taxa that are very common in grasslands. However, they also represent plants that develop in humid environments and near water bodies, such as Alternanthera philoxeroides and Ranunculus spp. Other herbs that also develop there are Polygonum punctatum, Nymphoides indica, Myriophyllum aquaticum and $M$. quitense, and so their pollen grains (Polygonum, Nymphoides indicA and Myriophyllum) were all found in the fossil assemblages. These associations represent freshwater areas that locally developed during the Holocene, but also suggest the occurrence of runoff that transported pollen from the hinterland source area to the estuary (Fig. 8). Relatively high ERYNGIUM proportions in the fossil sequences reflect the Eryngium pandanifolium dense clumps that frequently occur in wetlands (PB-IM) 
A) Arroyo Solís Grande (ASG)

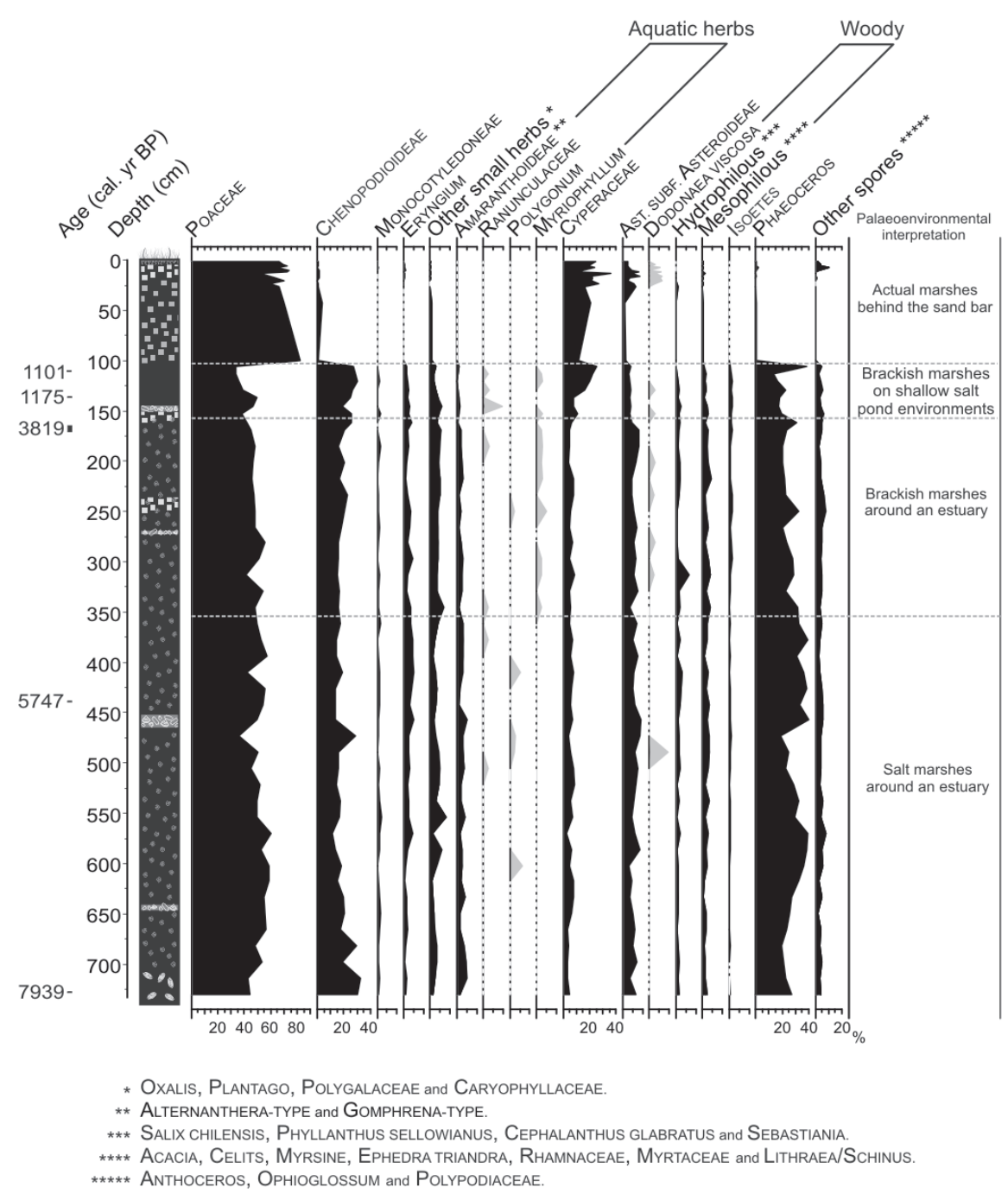

B) Paso Barranca-India Muerta (PB-IM)

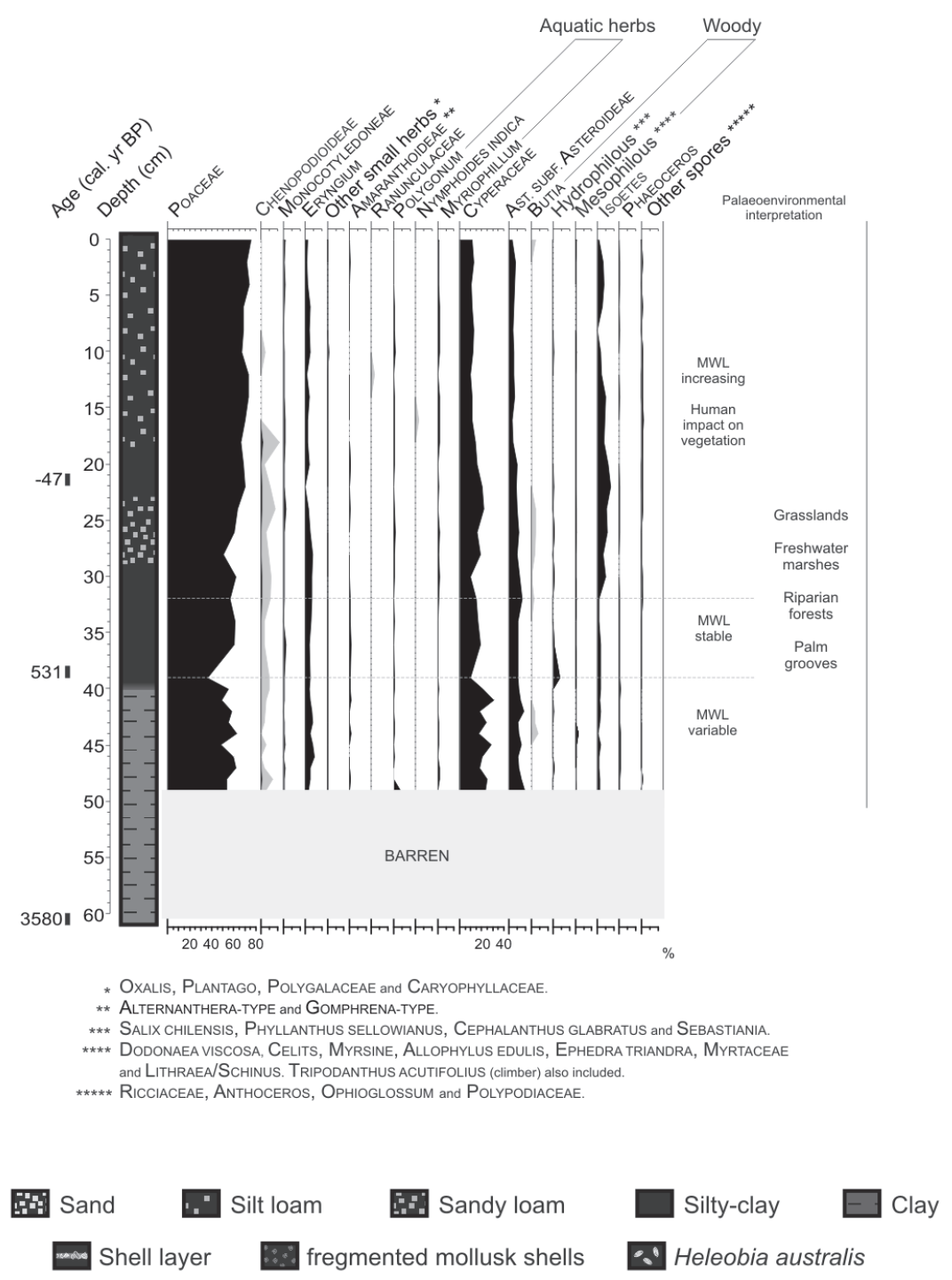

Figure 8. Calibrated ages, sediment description, percentage palynomorph diagrams and paleoenvironmental interpretation from (A) Arroyo Solís Grande (Mourelle et al. 2015a) and (B) Paso Barranca-India Muerta (Mourelle et al. 2015b) sequences, plotted against depth. MWL: marsh water level. Exaggeration 10x. 
and coastal areas (ASG) (Fig. 8).

The occurrence of certain pollen types in the fossil spectra, even in low proportions, turns out to be good indicators of paleoenvironmental changes. For instance, the appearance of the low salinity-tolerant MYRIOPHYLLUM in ASG sequence at around ca. 5100 cal. yr BP reflect an increase in freshwater input and a decrease in salinity derived from the transition from brackish to freshwater conditions during sea-level fall (Fig. 8). At the same time, DoDONAEA VISCOSA grains also appeared, indicating the development of psammophilous coastal vegetation in coastal sand bars (Fig. 8). On the contrary, ButiA pollen was found in low proportions and only in some PB-IM samples, disappearing towards the top of the core (Fig. 8). This initially suggests the development of palms interspersed among grasslands, close to the sampled site, at least since ca. 1300 cal. yr BP. However, as BuTIA pollen has not been found in all surface samples where palm individuals develop, its absence in the fossil assemblages prevents us to interpret this as an absence of palms in the past vegetation. Nevertheless, BUTIA disappearance in PB-IM assemblages corresponds to the last decades and probably reflects the palm vegetation decline due to human activities, such as pasture burning. This practice also reduces Eryngium pandanifolium clumps and Eupatorium spp. and Baccharis spp. shrubs, and this was also observed in the fossil spectra (Fig. 8).

Regarding the woody taxa, pollen from hydrophilous and mesophilous taxa were registered in the fossil sequences (Fig. 8). Key pollen types from hydrophilous taxa suggest the development of riparian forests. However, it is not possible to distinguish what type of forest or shrubland the pollen of mesophilous taxa comes from, since these plants are shared by all vegetation types. The knowledge of the geomorphology of the region (e.g. the proximity of the study area to hills) and its evolution (e.g. coastal progradation) is indispensable to determine if any of these forests or shrublands could have developed nearby in the past.

High spores proportions, mainly PHAEOCEROS, suggest the occurrence of runoff transported from the hinterland source area to the estuary in ASG, as these spores were not recorded in surface samples from coastal vegetation (Fig. 8). Very low proportions of deteriorated pollen grains and high pollen sum in the fossil spectra allowed us to rule out an over-representation of bryophyte spores due to poor preservation, which in addition amplifies the imperative value of the spores account. IsOETES relatively high proportions in PB-IM pollen assemblages probably reflect the edaphic characteristic of this wetland area that is usually covered by water.

\section{Conclusions}

This is the first catalogue that describes and illustrates pollen and spores in modern and fossil samples from the campos region of Uruguay. Distinctive palynological assemblages characterized the main vegetation types, mainly related to the dominance of some taxa (e.g. POACEAE in grasslands, DodonAEA VIScosA in "Candela" coastal shrublands) or to the presence of key indicator taxa (e.g. hydrophilous woody taxa in riparian forests). However, the low taxonomic resolution of some pollen taxa (e.g. Asteraceae subf. Asteroideae, Myrtaceae, RHAMNACEAE) and the absence of pollen from species that develop exclusively in some vegetation types (e.g. Sideroxylon obtusifolium, Rollinia maritima and Varronia curassavica in psammophilous forests; Juncus acutus in brackish marshes) makes it difficult to differentiate some vegetation types only by the palynological assemblages. The latter are considered silent taxa, and their identification proved to be very important in order not to misinterpret the fossil pollen spectra.

The knowledge of pollination strategies of parental plants was necessary to explain the presence of small amounts of key pollen types that result indicative of the vegetation (e.g. hydrophilous woody taxa indicate riparian forests development, aquatic herbs indicate freshwater areas, and BuTIA indicates palms development grasslands).

Studies like this one help and guide the palynologist to make identifications in surface or fossil samples, and thereby they are useful to focus on the taxa most likely to occur in the fossil record. It presents a practical approach to paleoecological research, which not only produces robust results in studies of the campos region, but can also be applied to other grassland ecosystems, including those in temperate regions.

\section{Acknowledgements}

This work is part of the PhD Thesis of one of the authors (D.M.) financially supported by two postgraduate scholarships (CONICET, Argentina). Funding was provided by CONICET (grants PIP 1265/09 and PIP 543/12); Universidad Nacional de Mar del Plata (grants Exa 150/10 and Exa 602/12); FONCYT (grant PICT 950/14); ANII, PEDECIBA and PDU-Geociencias CURE-Rocha (Uruguay). We thank F. García-Rodríguez for the linguistic revision; and R.B. Macedo and to two anonymous reviewers that greatly improved the final version of the manuscript.

\section{References}

Adam DP, Mehringer PJJ. 1975. Modern pollen surface samples. An analysis of subsamples. Journal Research U.S. Geological Survey 3: 733-736.

Alonso-Paz E, Bassagoda MJ. 1999. Los bosques y los matorrales psamófilos en el litoral platense y atlántico del Uruguay. Comunicaciones Botánicas del Museo de Historia Natural de Montevideo 6: 1-8.

Alonso-Paz E, Bassagoda MJ. 2002. Aspectos fitogeográficos y diversidad biológica de las formaciones boscosas del Uruguay. Ciência \& Ambiente 24: 35-50.

APG III. 2009. An update of the Angiosperm Phylogeny Group classification for the orders and families of flowering plants: APG III. Botanical Journal of the Linnean Society 161: 105-121. 
Bartesaghi ML. 2007. Análisis espacial de las formaciones vegetales costeras Matorral y Bosque, de la zona El Caracol, Departamento de Rocha, Uruguay. Graduate Thesis, Universidad de la República, Uruguay.

Bauermann SG, Radaeski JN, Evaldt ACP, et al. 2013. Pólen nas Angiospermas: diversidade e evolução. Rio Grande do Sul, Editora da ULBRA.

Behling H, Negrelle RRB. 2006. Vegetation and Pollen Rain Relationship from the Tropical Atlantic Rain Forest in Southern Brazil. Brazilian Archives of Biology and Technology 49: 631-642.

Bilenca D, Miñarro F. 2004. Identificación de Áreas Valiosas de Pastizal (AVPs) en las Pampas y Campos de Argentina, Uruguay y sur de Brasil. Buenos Aires, Fundación Vida Silvestre Argentina.

Birks HJB, Birks HH. 1980. Quaternary palaeoecology. London, Edward Arnold.

Birks HJB, Gordon AD. 1985. Numerical methods in Quaternary pollen analysis. London, Academic Press Inc.

Brussa CA, Grela I. 2007. Flora Arbórea del Uruguay. Con énfasis en las especies de Rivera y Tacuarembó. Montevideo, COFUSA.

Bush MB. 1991. Modern pollen-rain data from South and Central America: a test of the feasibility of fine-resolution lowland tropical palynology. The Holocene 1: 162-167.

Bush MB, Rivera R. 2001. Reproductive ecology and pollen representation among neotropical trees. Global Ecology and Biogeography Letters 10: 359-367.

Bush MB, Moreno E, Oliveira PE, Asanza E, Colinvaux PA. 2001. The influence of biogeographic and ecological heterogeneity on Amazonian pollen spectra. Journal of Tropical Ecology 17: 729-743.

Cancelli RR, Evaldt ACP, Bauermann SG, Souza PA, Bordignon SAL, Matzenbacher NI. 2010. Catálogo palinológico de táxons da família Asteraceae Martinov, no Rio Grande do Sul, Brasil. Iheringia, Série Botânica 65: 201-280.

Chebataroff J. 1960. Tierra Uruguaya. Montevideo, Talleres Don Bosco.

Erdtman G. 1971. Pollen morphology and plant taxonomy. Angiosperms (An Introduction to Playnology I). Stockholm, Almquist and Wiksell.

Evaldt ACP, Bauermann SG, Souza PA. 2013. Descrições morfológicas de palinomorfos holocênicos de um fragmento da Savana Estépica Parque em Barra do Quaraí, Rio Grande do Sul, Brasil. Pesquisas em Geociências 40: 209-232.

Fægri K, Iversen J. 1989. Textbook of Pollen Analysis. New York, John Wiley \& Sons.

Fagúndez C, Lezama F. 2005. Distribución Espacial de la Vegetación Costera del Litoral Platense y Atlántico Uruguayo. Montevideo, FREPLATA.

García-Rodríguez F, Stutz S, Inda H, Puerto L, Bracco R, Panario D. 2010. A multiproxy approach to inferring Holocene paleobotanical changes linked to sea-level variation, paleosalinity levels, and shallow lake alternative states in Negra Lagoon, SE Uruguay. Hydrobiologia 646: $5-20$.

Gassmann MI, Pérez CF. 2006. Trajectories associated to regional and extraregional pollen transport in the southeast of Buenos Aires province, Mar del Plata (Argentina). International Journal of Biometeorology 50: 280-291.

Grimm EC. 2004. Tilia Software 2.0.4. Illinois State Museum. Research and Collection Center. Springfield, Illinois.

Haretche F, Mai P, Brazeiro A. 2012. Woody flora of Uruguay: inventory and implication within the Pampean region. Acta Botanica Brasilica 26: 537-552.

Hesse M, Zetter R, Halbritter H, et al. 2009. Pollen Terminology. An illustrated Handbook. Vienna, Springer Verlag.

Hidalgo N. 2008. Aprovechamiento de néctar y flores de Erythrina (Fabaceae) por varias especies de aves. VII Congreso Nacional de Ornitología. Piura. p. 107.

Iriarte J. 2006. Vegetation and climate change since $14.810{ }^{14} \mathrm{C}$ yr. B.P. in southeastern Uruguay and implications for the rise of early Formative societies. Quaternary Research 65: 20-32.

Isacch JP, Costa CSB, Rodríguez-Gallego L, et al. 2006. Distribution of saltmarsh plant communities associated with environmental factors along a latitudinal gradient on the south-west Atlantic coast. Journal of Biogeography 33: 888-900.
Jackson ST. 2007. Looking forward from the past: history, ecology, and conservation. Frontiers in Ecology and the Environment 455.

Jan F, Schüler L, Behling H. 2015. Trends of pollen grain size variation in C3 and C4 Poaceae species using pollen morphology for future assessment of grassland ecosystem dynamics. Grana 54: 129-145.

Klerk P, Joosten H. 2007. The difference between pollen types and plant taxa: a plea for clarity and scientific freedom. Quaternary Science Journal 56: 162-171.

León RJC. 1991. Río de la Plata grasslands. In: Coupland RT. (ed.) Natural grasslands: introduction and western hemisphere. Ecosystems of the World. Amsterdam, Elsevier. p. 369-376, 380-387.

Lezama F, Altesor A, Pereira M, Paruelo JM. 2011. Capítulo I. Descripción de la heterogeneidad florística de los pastizales naturales de las principales regiones geomorgológicas de Uruguay. In: Altesor A, Ayala W, Paruelo JM. (eds.) Bases ecológicas y tecnológicas para el manejo de pastizales. Montevideo, Serie FPTA- INIA. p. 15-32.

Macedo RB, Souza PA, Bauermann SG. 2009. Catálogo de pólens, esporos e demais palinomorfos em sedimentos holocênicos de Santo Antônio da Patrulha, Rio Grande do Sul, Brasil. Iheringia, Série Botânica 64: 43-78.

Markgraf V, D’Antoni HL. 1978. Pollen Flora of Argentina. Tucson, The University of Arizona Press.

Masetto E, Lorscheitter ML. 2014. Palynomorphs in Holocene sediments from a paleolagoon in the coastal plain of extreme southern Brazil. Acta Botanica Brasilica 28: 165-175.

MOBOT - Missouri Botanical Garden. 2016. Electronic Database. <http:// www.tropicos.org> 10 May 2016.

Mourelle D. 2015. Cambios de la vegetación de la región de los campos de Uruguay en respuesta a diferentes forzantes durante el Holoceno. PhD Thesis, Universidad Nacional de Mar del Plata, Argentina.

Mourelle D, Prieto AR. 2012. Modern pollen assemblages of surface samples and their relationships to vegetation in the campos region of Uruguay. Review of Palaeobotany and Palynology 181: 22-33.

Mourelle D, Prieto AR, Pérez L, García-Rodríguez F, Borel CM. 2015a. Mid and late Holocene multiproxy analysis of environmental changes linked to sea-level fluctuation and climate variability of the Río de la Plata estuary. Palaeogeography, Palaeoclimatology, Palaeoecology 421: 75-88.

Mourelle D, Prieto AR, García-Rodríguez F. 2015b. Cambios de la vegetación en la cuenca de la Laguna Merín, Uruguay, durante los últimos ca. 2000 cal. años AP. Revista Brasileira de Paleontologia 18: 509-520.

Neves PCP, Bauermann SG. 2004. Catálogo Palinológico de cobserturas quaternárias no estado de Rio Grande do Sul (Guaíba e Capão do Leão), Brasil. Descrições taxonômicas - Parte II: Bryophyta e Pteridophyta. Pesquisas. Botânica 55: 227-251.

Ortuño T, Ledru MP, Cheddadi R, Kuentz A, Favier C, Beck S. 2011. Modern pollen rain, vegetation and climate in Bolivian ecoregions. Review of Palaeobotany and Palynology 165: 61-74.

Otegui M, Cocucci A. 1999. Flower morphology and biology of Myrsine Iaetevirens, structural and evolutionary implications of anemophily in Myrsinaceae. Nordic Journal of Botany 19: 71-85.

Overpeck JT, Webb TI, Prentice IC. 1985. Quantitative interpretation of fossil pollen Spectra: dissimilarity coefficients and the method of modern analogs. Quaternary Research 23: 87-108.

Petraglia C, Dell'Acqua M. 2006. Actualización de la carta forestal del Uruguay con imágenes del año 2004. Montevideo. p. 27.

Pire SM, Anzótegui LM, Cuadrado GA. 1998. Flora Polínica del Nordeste Argentino. Corrientes, EUDENE - UNNE.

Pire SM, Anzótegui LM, Cuadrado GA. 2001. Flora Polínica del Nordeste Argentino. Corrientes, EUDENE - UNNE.

Pire SM, Anzótegui LM, Cuadrado GA. 2006. Flora Polínica del Nordeste Argentino. Corrientes, EUDENE - UNNE.

Prentice IC. 1988. Records of vegetation in time and space: the principles of pollen analysis. In: Huntley B, Webb TI. (eds.) Vegetation History. Dordrecht, Kluwer Academic. p. 17-42.

Prieto AR, Quattrocchio ME. 1993. Briofitas y Pteridofitas en sedimentos del Holoceno de la provincia de Buenos Aires, Argentina. Anales de la Asociación de Palinólogos de Lengua Española 6: 17-37.

Prieto AR, Mourelle D, Peltier WR, Drummond R, Vilanova I, Ricci L. 2016. Relative sea-level changes during the Holocene in the Río de 
la Plata, Argentina and Uruguay: A review. Quaternary Internacional (in press). doi: 10.1016/j.quaint.2016.02.044.

Puerto O. 1969. Hierbas de Uruguay. Montevideo, Nuestra Tierra.

Puerto O. 1987. Vegetación del Uruguay. Montevideo, Facultad de Agronomía.

Punt W, Hoen PP. 2009. The Northwest European Pollen Flora, 70. Asteraceae - Asteroideae. Review of Palaeobotany and Palynology 157: 22-183.

Punt W, Hoen PP, Blackmore S, Nilsson S, Le Thomas A. 2007. Glossary of pollen and spore terminology. Rewiew of Paleobotany and Palynology 143: 1-91.

Radaeski JN. 2014. Morfologia polínica de táxons de Poaceae do Rio Grande do Sul: Uma abordagem para distinguir vegetações campestres e florestais no sul do Brasil. MSc Thesis, Universidade Federal do Pampa, Brazil.

Radaeski JN, Evaldt ACP, Bauermann SG, Lima GL. 2014. Diversidade de grãos de pólen e esporos dos Campos do sul do Brasil: descrições morfológicas e implicações paleoecológicas. Iheringia, Série Botânica 69: 107-132.

Rodgers III JC, Horn SP. 1996. Modern pollen spectra from Costa Rica. Palaeogeography, Palaeoclimatology, Palaeoecology 124: 53-71.

Rodríguez-Gallego L, Masciadri S, Nin M. 2012. Modern vegetation and pollen relationships in four southwestern atlantic coastal lagoons. Estuaries and Coasts 35: 785-798.

Rull V. 2010. Ecology and Palaeoecology: two approaches, one objective. The Open Ecology Journal 3: 1-5.
Schüler L, Behling H. 2011. Poaceae pollen grain size as a tool to distinguish past grasslands in South America: a new methodological approach. Vegetation History and Archaeobotany 20: 83-96.

Seppä H. 2007. Pollen Analysis, Principles. In: Elias S. (ed.) Encyclopedia of Quaternary Science. Amsterdam, Elsevier. p. 2486-2497.

Soriano A. 1991. Río de la Plata grasslands. In: Coupland RT. (ed.) Natural grasslands: introduction and western hemisphere. Ecosystems of the World. Amsterdam, Elsevier. p. 367-369.

Stutz S, Prieto AR. 2003. Modern pollen and vegetation relationships in Mar Chiquita coastal lagoon area, southeastern Pampa grasslands, Argentina. Review of Palaeobotany and Palynology 126: 183-195.

Tonello MS, Prieto AR. 2008. Modern vegetation-pollen-climate relationships for the Pampa grasslands of Argentina. Journal of Biogeography 35: 926-938.

Torretta JP, Basilio AM. 2009. Pollen dispersion and reproductive success of four tree species of a xerophytic forest from Argentina. Revista de Biologia Tropical 57: 283-292.

Vilanova I, Prieto AR. 2012. Historia de la vegetación de las llanuras costeras de la Bahía Samborombón $\left(\sim 35,5^{\circ} \mathrm{S}\right)$, Argentina, desde 7800 ${ }^{14} \mathrm{C}$ años. Ameghiniana 49: 303-318.

Villarreal JC, Cargill DC, Hagborg A, Söderström L, Renzaglia KS. 2010. A synthesis of hornwort diversity: Patterns, causes and future work. Phytotaxa 9: 150-166.

Willis KJ, Bailey RM, Bhagwat SA, Birks HJB. 2010. Biodiversity baselines, thresholds and resilience: testing predictions and assumptions using palaeoecological data. Trends in Ecology \& Evolution 25: 583-591. 